\title{
Stochastic Integration on the Full Fock Space with the Help of a Kernel Calculus
}

\author{
By \\ Roland SPEICHER*
}

\begin{abstract}
We develop a stochastic integration theory with respect to creation, annihilation and gauge operators on the full Fock space. This is done by using a kernel representation for a large class of bounded operators on the full Fock space. It is shown that the kernels form a Banach algebra. Having established the definition of processes and stochastic integrals we go on to prove an Ito formula and use this for examining stochastic evolutions and constructing dilations of special completely positive semigroups. Explicit solutions of the corresponding stochastic differential equations are given.
\end{abstract}

\section{$\S 1$. Introduction}

In non-commutative probability theory (quantum stochastics) one tries to develop a stochastic description of quantum systems. One of the first tasks is to develop a concept of white noise (as a model for a quantum heat bath) and to develop a stochastic integration with respect to this white noise in order to couple it via Langevin equations to other quantum systems. (cf. [Küm2, 3, Maa2])

Replacing the classical concepts of random variables and probability measures by the functional analytic concepts of operators and states, the characterization of 'white noise' will read in the following way. (cf. e.g. [AFL, Küm3, Spe2])

Definition. Let $\mathscr{R}$ be the ring generated by all semiclosed intervals $[s, t)$ $\subset \boldsymbol{R}$. An $n$-dimensional white noise is a quantum stochastic process $\left(\left(j_{I}\right)_{I \in \mathscr{R}}, \mathcal{A}, \mathscr{C}, \rho\right)$ in the sense of [AFL] with

$$
j_{I}:\left(\mathcal{A}, \rho_{I}\right) \longrightarrow(\mathscr{E}, \rho), \quad \rho_{I}=\rho \circ j_{I},
$$

Communicated by H. Araki, May 22, 1990.

* Institut für Angewandte Mathematik, Universität Heidelberg, Im Neuenheimer Feld 294, 6900 Heidelberg, Federal Republic of Germany.

I wish to thank Professor Wilhelm von Waldenfels and especially Burkhard Kümmerer for many interesting discussions. This work was supported by the Deutsche Forschungsgemeinschaft (SFB 123). 
where $A$ is the free $*$-algebra with $n$ generators $x_{1}, \cdots, x_{n}$, such that:

i) $j_{I_{1} \cup I_{2}}\left(x_{i}\right)=j_{I_{1}}\left(x_{\imath}\right)+j_{I_{2}}\left(x_{\imath}\right)$ for $i=1, \cdots, n$ and disjoint $I_{1}, I_{2} \in \mathscr{R}$.

ii) the distribution of the white noise is stationary, i. e. $\rho_{I}$ depends only on $\lambda(I)$, the Lebesgue measure of $I$.

iii) $j_{I_{1}}(A), j_{I_{2}}(A), \cdots, j_{I_{r}}(\mathcal{A})$ are independent for $r \in \mathbb{N}$ and disjoint $I_{1}, I_{2}, \cdots$, $I_{r} \in R$

But whereas the meaning of independence is clear in the classical case, we have some freedom in the non-commutative case: Let

$$
a_{1}:=j_{I_{1}}(a), \quad a_{2}:=j_{I_{2}}(a) \quad \text { with } a \in \mathcal{A} \text { and } I_{1}<I_{2},
$$

where $I_{1}<I_{2}$ means: $i_{1}<i_{2}$ for all $i_{1} \in I_{1}, i_{2} \in I_{2}$.

Then Kümmerer [Küm3] demands in his theory of 'generalized white noise' a factorization for time-ordered products, e.g. $\rho\left(a_{1} a_{1} a_{2} a_{2}\right)=\rho\left(a_{1} a_{1}\right) \rho\left(a_{2} a_{2}\right)$, but no apriori rules for calculating expressions like $\rho\left(a_{1} a_{2} a_{1} a_{2}\right)$ are given. Adding different rules for these expressions leads to different forms of independence and thus to different classes of white noises. Until now only two forms of independence have been used:

i) the bosonic case: Elements from independent algebras commute. According to the work of Schürmann [Sch] we know that we can realize the corresponding bosonic white noises with the help of creation, annihilation and gauge operators on a symmetric Fock space.

ii) the fermionic case: Elements from independent algebras anticommute (given a graduation). This leads to white noises which can be realized with the help of creation, annihilation and gauge operators on an antisymmetric Fock space. ([Sch])

The corresponding stochastic calculi were developed by Barnett, Streater, Wilde [BSW1, 2] and in an extended version (including Ito's formula) by Hudson and Parthasarathy [HuP1] and by Applebaum and Hudson [ApH]. It should be noted that the bosonic and fermionic calculi are essentially equivalent (see [HuP2]), although the corresponding white noises are of course distinct.

Voiculescu [Voi] introduced another form of independence, called 'free' independence. In [Spe2] we examined this free independence from a quantum stochastic point of view. In particular, we started to show that all free white noises can be realized with the help of creation, annihilation and gauge operators on a full Fock space. A complete treatment of this will follow in [GSS]. Thus we are left with the task to develop a stochastic calculus for these operators in order to couple free white noises as heat baths to other systems, i. e., to give meaning to Langevin equations with respect to free white noises.

While there is some hope that the bosonic and fermionic white noises may be of some use for describing physical situations, at least after taking some (weak coupling or singular coupling) limit (cf. [Dav, Düm, FGo, GaC]), the 
physical significance of free white noises is yet unclear. In this context it may be interesting to note that the free analogue of the Gaussian distribution gives the distribution of large random matrices with respect to the trace (see e.g. [Wig, Arn, Voi2]) and that the latter were used by Wigner [Wig] for modelling complex physical systems like heavy nuclei.

The stochastic calculus on the full Fock space was developed in [Spe1]. The stochastic calculus for the creation and annihilation processes will be presented in $[\mathrm{KSp}]$. The inclusion of the gauge process in this treatment presents some problems. Thus we redeveloped the whole theory in terms of a kernel representation, which allows the inclusion of the gauge process in a canonical way. This theory will be presented here. This kernel method is formally analogous to the corresponding method for the bosonic case, which was developed by Maassen [Maa1]. We want to stress the difference, that we describe with the kernels bounded operators on the full Fock space instead of unbounded operators on the symmetric Fock space. This allows us to consider the space of the kernels as a Banach algebra of bounded operators. In the bosonic case we can only deal with an algebra of unbounded operators. Furthermore, in our case the kernel representation is not only a nice reformulation of the existing theory of $[\mathrm{KSp}]$, but it is essential for dealing with the gauge process.

First we will make some conventions used in the following (see e.g. [DiU]): For a Banach space $X$ and a finite measure space $(\Gamma, \Sigma, \mu)$ we will denote by $L^{p}(\Gamma ; X)(1 \leqq p<\infty)$ the Banach space of all equivalence classes of $X$-valued, Bochner-integrable functions $f: \Gamma \rightarrow X$, equipped with the norm

$$
\|f\|_{p}:=\left(\int_{\Gamma}\|f(\omega)\|^{p} d \mu(\omega)\right)^{1 / p} .
$$

We recall that the simple functions (i.e. the functions with finite range) are dense in $L^{p}(\Gamma ; X)$. For $p=\infty$ we denote by $L^{\infty}(\Gamma ; X)$ the Banach space of all equivalence classes of $X$-valued, Bochner-integrable functions $f: \Gamma \rightarrow X$, equipped with the norm

$$
\|f\|_{\infty}:=\operatorname{ess} \sup \{\|f(\omega)\| \mid \omega \in \Gamma\} .
$$

We recall that functions with countable range are dense in $L^{\infty}(\Gamma ; X)$.

By $\mathfrak{L}\left(\boldsymbol{R}^{n}\right)$ we denote the Borel subsets of $\boldsymbol{R}^{n}$ and by $\chi_{A}$ the characteristic function for $A \in \mathfrak{L}(\boldsymbol{R})$.

The paper is organized as follows. In Section 2 we recall the definition and some fundamental facts about the full Fock space and the creation, annihilation and gauge operators. We also outline the main ideas of the kernel representation. In Section 3 we define the kernels and their corresponding operators and show that the set of kernels forms a Banach algebra. We go on to define processes (Section 4) and stochastic integrals of adapted processes with respect to 
creation, annihilation and gauge process (Section 5). In Section 6 we establish an Ito's formula. Having shown the existence and uniqueness of the solution of linear stochastic differential equations (Section 7) we use Ito's formula in Section 8 to derive necessary and sufficient conditions on the coefficients of stochastic differential equations for the unitarity of the solution. Such unitary solutions are used in Section 9 for constructing dilations of special completely positive semigroups. Our kernel representation allows us to give explicit solutions of the differential equations.

\section{$\S 2$. Some Basic Facts about the Full Fock Space}

Let $\mathscr{K}_{0}$ be a Hilbert space. Then the non-symmetrized or full Fock space of $\mathscr{H}_{0}$ is the Hilbert space

$$
\mathscr{I}\left(\mathscr{H}_{0}\right):=C \Omega \oplus \bigoplus_{n=1}^{\infty} \mathcal{H}_{0}^{\otimes n},
$$

where $\Omega$ is a fixed unit vector. Hence the scalar product is given by $\left(f_{\imath}, g_{\imath}\right.$ $\left.\in \mathscr{H}_{0}\right)$

$$
\begin{gathered}
\left\langle f_{1} \otimes \cdots \otimes f_{n}, g_{1} \otimes \cdots \otimes g_{m}\right\rangle=\delta_{n m}\left\langle f_{1}, g_{1}\right\rangle \cdots\left\langle f_{n}, g_{n}\right\rangle \\
\left\langle\Omega, f_{1} \otimes \cdots \otimes f_{n}\right\rangle=0 \\
\langle\Omega, \Omega\rangle=1 .
\end{gathered}
$$

For each $f \in \mathcal{H}_{0}$ we define the left annihilation operator $l(f)$ and the left creation operator $l^{*}(f)$ by

$$
\begin{aligned}
& l(f) f_{1} \otimes \cdots \otimes f_{n}=\left\langle f, f_{1}\right\rangle f_{2} \otimes \cdots \otimes f_{n} \\
& l^{*}(f) f_{1} \otimes \cdots \otimes f_{n}=f \otimes f_{1} \otimes \cdots \otimes f_{n} .
\end{aligned}
$$

The operators $l(f)$ and $l^{*}(f)$ are bounded and mutually adjoint. Furthermore

$$
\|l(f)\|=\left\|l^{*}(f)\right\|=\|f\|_{\mathscr{H}_{0}} .
$$

We will also write: $l(A):=l\left(\chi_{A}\right)$ for $A \in \mathfrak{L}(\mathbb{R})$.

We will only consider $\mathscr{A}_{0}=L^{2}(\mathbb{R})$ and denote $\mathscr{I}\left(L^{2}(\mathbb{R})\right)$ by $\mathscr{F}$.

For $I \in \mathbb{L}(\mathbb{R})$ we define

$$
O(I):=C^{*}\left(l(f) \mid f \in L^{2}(I)\right) \subset B(\mathscr{F})
$$

as the $C^{*}$-algebra generated by all annihilation operators adapted to $I$. Then $O(I)$ is as a $C^{*}$-algebra isomorphic to the Cuntz algebra $O_{\infty}$ [Cun, Eva]. It is characterized by the relations

$$
l(f) l^{*}(g)=\langle f, g\rangle 1 \quad \text { for } f, g \in L^{2}(I) .
$$

It is important to note that because of these relations we can write a product of creation and annihilation operators in the following standard form: 


$$
l *\left(f_{1}\right) \cdots l *\left(f_{n}\right) l\left(g_{1}\right) \cdots l\left(g_{l}\right),
$$

with $n, l \in \mathbb{N}_{0}, f_{i}, g_{\imath} \in L^{2}(I)$.

Furthermore we define gauge operators analogously to the bosonic case (cf. [HuP1]). For each $T \in B\left(L^{2}(\boldsymbol{R})\right)$ we define the operator $p(T) \in B(\mathscr{T})$ by :

We have

$$
\begin{gathered}
p(T) \Omega=0 \\
p(T) f_{1} \otimes f_{2} \otimes \cdots \otimes f_{n}=\left(T f_{1}\right) \otimes f_{2} \otimes \cdots \otimes f_{n} .
\end{gathered}
$$

$$
\|p(T)\|_{B(\mathscr{F})}=\|T\|_{B\left(L^{2}(R)\right)} .
$$

If $T$ is compact then $p(T)$ is an element of $O_{\infty}$, because $T$ may be approximated by operators $T_{n}$ which have finite rank. For such operators we have a representation of $p\left(T_{n}\right)$ by creation and annihilation operators: For example, for the operator of $\operatorname{rank} 1\left(f_{1}, g_{1} \in L^{2}(\mathbb{R})\right)$ :

$$
T=\left|f_{1}\right\rangle\left\langle g_{1}\right|: L^{2}(\boldsymbol{R}) \longrightarrow L^{2}(\boldsymbol{R}), f \longmapsto T f:=\left\langle g_{1}, f\right\rangle \cdot f_{1}
$$

we can write $p(T)$ as $p(T)=l *\left(f_{1}\right) l\left(g_{1}\right)$.

For $T, T_{1}, T_{2} \in B\left(L^{2}(\mathbb{R})\right), f \in L^{2}(\mathbb{R})$ we have the following additional relations :

$$
\begin{gathered}
p(T)^{*}=p\left(T^{*}\right) \\
p\left(T_{1}\right) p\left(T_{2}\right)=p\left(T_{1} T_{2}\right) \\
l(f) p(T)=l\left(T^{*} f\right) \\
p(T) l^{*}(f)=l *(T f) .
\end{gathered}
$$

We are especially interested in the multiplication operators $T_{h}$ defined by

$$
T_{h} f=h f,
$$

for $h \in L^{\infty}(R)$. We have

$$
\left\|p\left(T_{h}\right)\right\|_{B(\Phi)}=\|h\|_{L^{\infty}(R)} .
$$

We will also write $p(h):=p\left(T_{h}\right)$ and especially

$$
p(A):=p\left(\chi_{A}\right)=p\left(T_{\chi_{A}}\right)
$$

for $A \in \mathfrak{L}(\boldsymbol{R})$. Since subsets of measure zero play no role, we can write $p(s, t)$ $:=p([s, t])=p((s, t))=\cdots$.

Since multiplication operators are not compact, the operators $p(A)$ do not belong to the Cuntz algebra $O_{\infty}$ for $\lambda(A) \neq 0$.

We now want to define a stochastic integration, where the gauge process is allowed as an integrator too. Thus we want to give meaning to expressions like $\int G(t) d \bar{l}(t) F(t)$, where $d \bar{l}(t)$ denotes one of the three integrators $d l(t), d l^{*}(t)$ and of $d p(t)$, and $G$ and $F$ are processes from some time interval $K$ into a 
suitably chosen subspace of the bounded operators on the full Fock space. The appearence of two processes, one on each side of the differential $d \bar{l}(t)$, is forced by the absence of any commutation relation between freely independent subalgebras. Thus, even if $G, F$ are adapted, we have no chance to reduce the above general form to the usual one, namely to $\int H(t) d \bar{l}(t)$.

We could try developing this theory by copying our stochastic integration theory $[\mathrm{KSp}]$ for creation and annihilation process. Thus we would replace the $C^{*}$-algebras $O(I)$ by

$$
\hat{O}(I):=C^{*}\left(p(h), l(f) \mid f \in L^{2}(I), h \in L^{\infty}(I)\right) .
$$

But then we are at once confronted with the problem that we cannot define all needed integrals in the usual way. To see this consider: $F(t)=\int_{0}^{t} d p(t)=p(0, t)$. Since $p(A)$ is a non vanishing orthogonal projection for all $A \in \mathbb{R}(\mathbb{R})$ with $\lambda(A)$ $\neq 0$, we have for all $t \neq t^{\prime}:\left\|F(t)-F\left(t^{\prime}\right)\right\|=1$. Thus we can not approximate $F(t)$ uniformly by simple functions and the usual definition fails for expressions like

$$
M=\int_{I} F(t) d l(t)=\int_{I} p(0, t) d l(t) .
$$

Of course it is clear how we should try to define $M$ : Let, for each $r$, a partition of $I$ in $r$ disjoint subintervals $I_{r}^{k}=\left[t_{r}^{k}, t_{r}^{k+1}\right)(k=1, \cdots, r)$ of the same length be given. Then define

$$
M:=\lim _{r \rightarrow \infty} \sum_{k=1}^{r} p\left(0, t_{r}^{k}\right) l\left(I_{r}^{k}\right)
$$

We have to check in which topology this limit exists and whether it is independent of the chosen partition of $I$. By estimates for the norm it is possible to show that $M$ can be defined indeed as an element of $\hat{O}(\mathbb{R})$ in this way, and we could try to extend the theory of $[\mathrm{KSp}]$ in this way. Instead of following these lines we will present here a kernel calculus which gives a more elegant representation for a big class of operators on the Fock space and for the definition of stochastic integrals. In this way, it allows a self-contained solution of the above problem.

Because of the above algebraic relations for the elements of $\hat{O}(\mathbb{R})$, it is possible to write a product of creation, annihilation and gauge operators in the standard form

$$
l *\left(f_{1}\right) \cdots l *\left(f_{n}\right) p(h) l\left(g_{1}\right) \cdots l\left(g_{l}\right),
$$

with $n, l \in N_{0}, f_{i}, g_{i} \in L^{2}(\mathbb{R})$ and $h \in L^{\infty}(\boldsymbol{R})$ (where this form shall implicitly include the cases where no $l^{*}, p$ or $l$ appears). Thus every operator of $\hat{O}(\boldsymbol{R})$ can be approximated by sums of operators of the above form.

The idea of the kernel representation is to describe operators which have 
the above standard form by functions according to

$$
l^{*}\left(f_{1}\right) \cdots l *\left(f_{n}\right) p(h) l\left(g_{1}\right) \cdots l\left(g_{m}\right) \longleftrightarrow f:=f_{1} \otimes \cdots \otimes f_{n} \otimes h \otimes g_{1} \cdots \otimes g_{m} .
$$

In this case the kernel is a function on $\boldsymbol{R}^{n} \times \boldsymbol{R} \times \boldsymbol{R}^{m}$. If we now close the space of this functions in the 'right' norm ( $L^{\infty}$-norm for $p, L^{2}$-norm for $l$ and $l^{*}$ ), then each element of $\mathfrak{B}^{(n, 1, l)}:=L^{\infty}\left(\boldsymbol{R} ; L^{2}\left(\boldsymbol{R}^{n} \times \mathbb{R}^{l}\right)\right)$ corresponds to a bounded operator on the Fock space. Now consider processes from a time interval $K \subset \boldsymbol{R}$ to the space of kernels in $\mathfrak{B}^{(n, 1, l)}$. At the first sight we would define this by $L^{\infty}\left(K, \mathfrak{B} \mathfrak{B}^{(n, 1, l)}\right)=L^{\infty}\left(K ; L^{\infty}\left(\mathbb{R} ; L^{2}\left(\boldsymbol{R}^{n} \times \boldsymbol{R}^{l}\right)\right)\right)$. This corresponds to the usual definition as the closure of the simple functions and would face us with the problem mentioned at the beginning. But in this representation we can extend this space in a natural way to the larger space $L^{\infty}\left(K \times \boldsymbol{R} ; L^{2}\left(\boldsymbol{R}^{n} \times \boldsymbol{R}^{l}\right)\right)$. The difference lies mainly in the replacing of the $C^{*}$-tensor product $L^{\infty}(K) \otimes$ $L^{\infty}(\boldsymbol{R})$ by the $W^{*}$-tensor product $L^{\infty}(K) \bar{\otimes} L^{\infty}(\boldsymbol{R})=L^{\infty}(K \times \boldsymbol{R})$. In this way we extend the space of the allowed processes crucially-e.g. the above mentioned $F$ is an element of $L^{\infty}\left(K \times \boldsymbol{R} ; L^{2}\left(\boldsymbol{R}^{0} \times \boldsymbol{R}^{0}\right)\right)$, but not of $L^{\infty}\left(K ; L^{\infty}\left(\boldsymbol{R} ; L^{2}\left(\boldsymbol{R}^{0} \times \boldsymbol{R}^{0}\right)\right)\right.$ ). A further advantage of this representation is that the definition of stochastic integrals is possible in a uniform way for all allowed processes-not only for simple ones.

In view of our stochastic integration theory there is also another way to look at the kernels: the kernel $f\left(t_{1}, \cdots, t_{n} ; z ; s_{1}, \cdots, s_{l}\right)$ will correspond to the operator

$$
\int \cdots \int f\left(t_{1}, \cdots, t_{n} ; z ; s_{1}, \cdots, s_{l}\right) d l^{*}\left(t_{1}\right) \cdots d l^{*}\left(t_{n}\right) d p(z) d l\left(s_{1}\right) \cdots d l\left(s_{l}\right) .
$$

Thus our integration theory is a theory of iterated integrals and the definition of the kernels and their corresponding operators is just an explicit definition of these iterated integrals. With this in mind the definition of stochastic integrals in Section 5 might be more reasonable. This point of view may also be useful for a transfer of [LiP] to the full Fock space.

We also want to point out that one can realize $l^{*}(f), l(g)$ and $p(h)$ with the help of the bosonic creation, annihilation and gauge operators (see [PSi]), but that this isomorphism does not imply the equivalence of the corresponding stochastic calculi, since the notion of adaptedness is not respected.

\section{§ 3. Definition and Properties of Kernels}

We now want to consider kernels corresponding to operators on $\mathscr{T} \otimes \mathscr{F}_{\tilde{I}}$, where $\mathcal{H}$ is a separable Hilbert space ('initial space'). Then a kernel is a mapping

$$
\begin{gathered}
f: \Gamma \times \tilde{\boldsymbol{R}} \times \Gamma \longrightarrow B(\mathscr{H}) \\
(\sigma ; z ; \nu) \longmapsto f(\sigma ; z ; \nu),
\end{gathered}
$$


where $\tilde{\mathbb{R}}$ is the (measure theoretic) direct sum of $\mathbb{R}^{0}$ and $\mathbb{R}^{1}$, and $\Gamma$ is the direct sum of all $\mathbb{R}^{n}(n \geqq 0)$ : Let $\mathbb{R}^{0}:=\{\emptyset\}, \lambda^{0}:=\delta_{\not \varnothing}$. Let $\Sigma^{i}$ be the Borel- $\sigma$ algebra of $\mathbb{R}^{i}\left(\Sigma^{0}:=\left\{\emptyset, \mathbb{R}^{0}\right\}\right)$ and $\lambda^{i}$ the Lebesgue-measure of $\mathbb{R}^{i}$ restricted to a compact subset $K_{i} \subset \mathbb{R}^{i}$, e. g. we could choose $K_{i}=K^{i}$, where $K$ is the time interval appearing in the definition of processes.

Then define

$$
\begin{aligned}
& (\tilde{\mathbb{R}}, \tilde{\Sigma}, \tilde{\lambda}):=\bigoplus_{n=0,1}\left(\mathbb{R}^{n}, \Sigma^{n}, \lambda^{n}\right)=\left(\mathbb{R}^{0}, \Sigma^{0}, \lambda^{0}\right) \oplus\left(\mathbb{R}^{1}, \Sigma^{1}, \lambda^{1}\right) \\
& (\Gamma, \Sigma, \mu):=\bigoplus_{n \geqq 0}\left(\mathbb{R}^{n}, \Sigma^{n}, \lambda^{n}\right) .
\end{aligned}
$$

The restriction of the kernel $f$ to $\mathbb{R}^{n} \times \mathbb{R}^{r} \times \mathbb{R}^{l}\left(n, l \in \mathbb{N}_{0} ; r=0,1\right)$ will be denoted by $f^{(n, r, l)}$.

Thus we can write

$$
f=\underset{(n, r, l)}{\bigoplus^{(n, r, l)},}
$$

where the sum runs over all triples with $n, l \in \mathbb{N}_{0}$ and $r=0,1$.

All our essential considerations will be for fixed $(n, r, l)$, and then we will lift everything from the single summands $f^{(n, r, l)}$ to $f$ by taking an $l^{1}$-direct sum.

Defimition. The space of kernels is the Banach space

$$
\begin{aligned}
& \mathfrak{B}:=\underset{(n, r, l)}{\bigoplus} l_{1} \mathfrak{S}^{(n, r, l)} \\
& =\left\{f=\bigoplus_{(n, r, l)} f^{(n, r, l)} \mid f^{(n, r, l)} \in \mathfrak{B}(n, r, l) ;\|f\|_{\mathfrak{B}}:=\sum_{(n, r, l)}\left\|f^{(n, r, l)}\right\|<\infty\right\},
\end{aligned}
$$

where the Banach spaces $\mathfrak{B}^{(n, r, l)}$ are defined by

$$
\mathfrak{B}^{(n, r, l)}:=L^{\infty}\left(\mathbb{R}^{r} ; L^{2}\left(\mathbb{R}^{n} \times \mathbb{R}^{l} ; B(\mathscr{H})\right)\right) .
$$

The space $\mathfrak{B}^{(n, r, l)}$ may be considered as a subspace of the $B(\mathscr{H})$-valued functions on $\mathbb{R}^{n} \times \mathbb{R}^{r} \times \mathbb{R}^{l}$.

Thus the norm on $\mathfrak{B}^{(n, r, l)}$ is given by

$$
\left\|f^{(n, r, l)}\right\|^{2}=\sup _{z \in R^{r}} \iint\left\|f^{(n, r, l)}(\sigma ; z ; \nu)\right\|_{B(\mathscr{H})}^{2} d \lambda^{n}(\sigma) d \lambda^{l}(\nu),
$$

where sup denotes the essential supremum with respect to $\lambda^{r}$. As a set we can consider $\mathfrak{B}$ in a canonical way as a proper subset of $L^{\infty}\left(\tilde{\boldsymbol{R}} ; L^{2}(\Gamma \times \Gamma ; B(\mathscr{H}))\right)$.

We write now

and notice

$$
\begin{aligned}
\mathscr{F} & =\mathbb{C} \Omega \oplus \underset{n \geqq 1}{\bigoplus^{2}} L^{2}(\mathbb{R})^{\otimes n} \\
& \cong C \Omega \oplus \bigoplus_{n \geqq 1} L^{2}\left(\mathbb{R}^{n}\right) \\
& \cong L^{2}(\Gamma)
\end{aligned}
$$


where

$$
\mathscr{H} \otimes \mathscr{F}=\mathscr{H} \otimes L^{2}(\Gamma)=L^{2}(\Gamma ; \mathscr{K}),
$$

$$
\begin{aligned}
L^{2}(\Gamma ; \mathcal{H}) & =\left\{\xi: \Gamma \rightarrow \mathscr{H} \mu \text {-measurable; }\|\xi\|^{2}:=\int\|\xi(\omega)\|_{\mathscr{H}}^{2} d \mu(\omega)<\infty\right\} \\
& =\bigoplus_{n \geq 0} L^{2}\left(\boldsymbol{R}^{n} ; \mathscr{H}\right) .
\end{aligned}
$$

We make the following notational conventions: For $\sigma_{1}=\left(t_{1}, \cdots, t_{n}\right) \in \boldsymbol{R}^{n}$ and $\sigma_{2}=\left(s_{1}, \cdots, s_{k}\right) \in \boldsymbol{R}^{k}$, we define the connecting

and the transpose

$$
\sigma_{1} \sigma_{2}:=\left(t_{1}, \cdots, t_{n}, s_{1}, \cdots, s_{k}\right) \in \boldsymbol{R}^{n+k}
$$

$$
\sigma_{1}^{*}:=\left(t_{n}, \cdots, t_{1}\right) \in \mathbb{R}^{n} .
$$

In particular, we have $\sigma \emptyset=\emptyset \sigma=\sigma$. We will also often use the differential formula

$$
d \mu\left(\sigma_{1} \sigma_{2}\right)=d \mu\left(\sigma_{1}\right) d \mu\left(\sigma_{2}\right) .
$$

Furthermore, for $\sigma=\left(t_{1}, \cdots, t_{n}\right) \in \mathbb{R}^{n}$ we define:

$$
\begin{aligned}
& \max (\boldsymbol{\sigma}):=\max _{i=1, \cdots, n} t_{i} \quad(\max (\emptyset):=\emptyset) \\
& \operatorname{beg}(\sigma):=t_{1} \quad(\operatorname{beg}(\emptyset):=\emptyset) \\
& \text { end }(\sigma):=t_{n} \quad(\operatorname{end}(\emptyset):=\emptyset) \text {, }
\end{aligned}
$$

and $\sigma \subset I$ with $I \in \mathbb{L}(\mathbb{R})$ stands for $\left\{t_{1}, \cdots, t_{n}\right\} \subset I$.

If $\omega \in \mathbb{R}^{s}$ is given, then

$$
\sum_{\omega_{1} z \omega_{2}=\omega}
$$

denotes the sum over all $\omega_{1}, z, \omega_{2}$ with $\omega_{1} \in \mathbb{R}^{l}, \omega_{2} \in \mathbb{R}^{k}(0 \leqq k, l \leqq s)$ and $z \in \mathbb{R}^{r}$ $(r=0,1)$ with $\omega_{1} z \omega_{2}=\omega$.

Now we map a kernel $f \in \mathfrak{B}$ to an operator $F$ on $\mathscr{H} \otimes \mathscr{F}=L^{2}(\Gamma ; \mathscr{H})$ in the following way:

$$
(F \xi)(\omega)=\sum_{\omega_{1} z \omega_{2}=\omega} \int f\left(\omega_{1} ; z ; \nu\right) \xi\left(\nu * z \omega_{2}\right) d \mu(\nu),
$$

where the integral is interpreted as the Bochner integral of the mapping

$$
\begin{gathered}
\Gamma \longrightarrow \mathscr{H} \\
\nu \longmapsto f\left(\omega_{1} ; z ; \nu\right) \xi\left(\nu^{*} z \omega_{2}\right) .
\end{gathered}
$$

Note that the sum reduces to one summand if $f=f^{(n, r, l)}$ for fixed $(n, r, l)$ :

$$
\left(F^{(n, r, l)} \xi\right)\left(\omega_{1} z \omega_{2}\right)=\int f^{(n, r, l)}\left(\omega_{1} ; z ; \nu\right) \xi\left(\nu * z \omega_{2}\right) d \lambda^{l}(\nu)
$$

for $\omega_{1} \in \mathbb{R}^{n}$ and $z \in \mathbb{R}^{r}$. 


\section{Eramples.}

1) $f=f^{(1,0,0)} \in \mathfrak{B}^{(1,0,0)}$ with $f(t ; 0 ; 0)=g(t) L$, where $g \in L^{2}(\mathbb{R})$ and $L \in B(\mathscr{H})$ Then

$$
(F \xi)(\omega)=\sum_{t \varnothing \omega_{2}=\omega} g(t) L \xi\left(\omega_{2}\right),
$$

which gives for $\xi=x \otimes f_{1} \otimes \cdots \otimes f_{n}$ with $f_{2} \in L^{2}(\mathbb{R})$ and $x \in \mathscr{H}$ (i. e. $\left.\xi\left(t_{1}, \cdots, t_{n}\right)=f_{1}\left(t_{1}\right) \cdots f_{n}\left(t_{n}\right) x\right)$ :

$$
\begin{aligned}
\left(F x \otimes f_{1} \otimes \cdots \otimes f_{n}\right)\left(t, t_{1}, \cdots, t_{n}\right) & =g(t) f_{1}\left(t_{1}\right) \cdots f_{n}\left(t_{n}\right) L x \\
& =\left(L x \otimes g \otimes f_{1} \otimes \cdots \otimes f_{n}\right)\left(t, t_{1}, \cdots, t_{n}\right),
\end{aligned}
$$

i. e. $F=L \otimes l^{*}(g)$.

2) $f=f^{(0,0,1)} \in \mathfrak{F}^{(0,0,1)}$ with $f(0 ; \emptyset ; s)=\bar{g}(s) L$, where $g \in L^{2}(\mathbb{R})$ and $L \in B(\mathscr{G C})$. Then we have

$$
(F \xi)(\omega)=\int f(\emptyset ; 0 ; s) \xi\left(s(\omega) d \lambda(s)=\int \bar{g}(s) L \xi(s \omega) d \lambda(s),\right.
$$

and thus $F=L \otimes l(g)$.

3) $f=f^{(0,1,0)} \in \mathfrak{Q}^{(0,1,0)}$ with $f(0 ; z ; \emptyset)=h(z) L$, where $h \in L^{\infty}(\mathbb{R})$ and $L \in B(\mathscr{A})$. Then we have

$$
(F \xi)(\omega)=\sum_{\delta z \omega_{2}=\omega} f(0 ; z ; 0) \xi\left(z \omega_{2}\right)=h(\operatorname{beg}(\omega)) L \xi(\omega),
$$

and thus $F=L \otimes p(h)$.

4) More generally the kernel $f=f^{(n, r, l)} \in \mathfrak{B}^{(n, r, l)}$ with

$$
f^{(n, r, l)}=f_{1} \otimes \cdots \otimes f_{n} \otimes h \otimes g_{1} \otimes \cdots \otimes g_{l} \cdot L
$$

( $f_{\imath}, g_{\imath} \in L^{2}(\mathbb{R}), h \in L^{\infty}(\mathbb{R}), L \in B(\mathscr{C})$ ) corresponds to the operator

$$
F=L \otimes l *\left(f_{1}\right) \cdots l^{*}\left(f_{n}\right) p(h) l\left(g_{1}\right) \cdots l\left(g_{l}\right) .
$$

5) The identity on $\mathscr{T} \otimes \mathscr{F}$ corresponds to the kernel id $=\mathrm{id}^{(0,0,0)} \in \mathfrak{B}^{(0,0,0)}$, given by $\operatorname{id}^{(0,0,0)}(\emptyset ; \emptyset ; \emptyset)=1_{B(\mathscr{C})}$, where $1_{B(\mathscr{A})}$ is the identity on $\mathscr{A}$.

Theorem $\mathbb{1}$. a) We have $\|F\| \leqq\|f\|_{\mathfrak{B}}$. In particular, $F \in B(\mathscr{H} \otimes \mathscr{F})$ for $f \in \mathfrak{B}$.

b) The mapping

$$
\begin{gathered}
\mathfrak{B} \longrightarrow B(\mathscr{H} \otimes \mathscr{F}) \\
f \longmapsto F
\end{gathered}
$$

is injective.

Proof. a) Let $F^{(n, r, l)}$ be the operator corresponding to $f^{(n, r, l)}$. Then $f=\bigoplus_{(n, r, l)} f^{(n, r, l)}$ implies $F=\sum_{(n, r, l)} F^{(n, r, l)}$. Thus it is suificient to show

$$
\left\|F^{(n, r, l)}\right\| \leqq\left\|f^{(n, r, l)}\right\|,
$$


because this gives at once

$$
\|F\|=\left\|\sum_{(n, r, l)} F^{(n, r, l)}\right\| \leqq \sum_{(n, r, l)}\left\|F^{(n, r, l)}\right\| \leqq \sum_{(n, r, l)}\left\|f^{(n, r, l)}\right\|=\|f\|_{\mathfrak{B}} .
$$

For proving the inequality let $\xi \in \mathscr{F}$.

$$
\begin{aligned}
& \left\|F^{(n, r, l)} \xi\right\|^{2}=\int\left\|\left(F^{(n, r, l)} \xi\right)(\omega)\right\|_{{ }^{2} l}^{2} d \mu(\omega) \\
& =\iiint \iint f^{(n, r, l)}\left(\omega_{1} ; z ; \nu\right) \xi\left(\nu^{*} z \omega_{2}\right) d \lambda^{l}(\nu) \|_{\mathscr{H}}^{2} d \lambda^{n}\left(\omega_{1}\right) d \lambda^{r}(z) d \mu\left(\omega_{2}\right) \\
& \stackrel{(*)}{\leqq} \iiint\left\{\int\left\|f^{(n, r, l)}\left(\omega_{1} ; z ; \nu\right)\right\|_{B(\mathscr{H})}^{2} d \lambda^{l}(\nu)\right\} \\
& \left\{\int\left\|\xi\left(\nu^{*} z \omega_{2}\right)\right\|_{\mathscr{H}}^{2} d \lambda^{l}(\nu)\right\} d \lambda^{n}\left(\omega_{1}\right) d \lambda^{r}(z) d \mu\left(\omega_{2}\right) \\
& =\iint\left\{\iint\left\|f^{(n, r, l)}\left(\omega_{1} ; z ; \nu\right)\right\|_{B(\mathscr{H})}^{2} d \lambda^{l}(\nu) d \lambda^{n}\left(\omega_{1}\right)\right\} \\
& \left\{\int\left\|\xi\left(\nu^{*} z \omega_{2}\right)\right\|_{\mathscr{H}}^{2} d \lambda^{l}(\nu)\right\} d \lambda^{r}(z) d \mu\left(\omega_{2}\right) \\
& \leqq\left\{\sup _{z^{\prime} \in R} \iint\left\|f^{(n, r, l)}\left(\omega_{1} ; z^{\prime} ; \nu\right)\right\|_{B(\mathscr{C})}^{2} d \lambda^{l}(\nu) d \lambda^{n}\left(\omega_{1}\right)\right\} \\
& \left\{\iiint\left\|\xi\left(\nu^{*} z \omega_{2}\right)\right\|_{\mathscr{H}}^{2} d \lambda^{l}(\nu) d \lambda^{r}(z) d \mu\left(\omega_{2}\right)\right\} \\
& \leqq\left\|f^{(n, r, l)}\right\|^{2}\|\xi\|^{2} \text {, }
\end{aligned}
$$

thus

$$
\left\|F^{(n, r, l)}\right\| \leqq\left\|f^{(n, r, l)}\right\| \text {. }
$$

In $(*)$ we have used the Cauchy-Schwarz-inequality for the integration with respect to $\nu$.

b) Consider

$$
F=\sum_{(n, r, l)} F^{(n, r, l)}=0 .
$$

We have to show that the corresponding kernel

$$
f=\bigoplus_{(n, r, l)} f^{(n, r, l)}
$$

vanishes.

We write $F: L^{2}(\Gamma ; \mathscr{H}) \rightarrow L^{2}(\Gamma ; \mathscr{H})$ as a matrix

$$
F=\left(F^{[m, n]}\right)_{n, m \in N_{0}}: \bigoplus_{n \geq 0} L^{2}\left(\mathbb{R}^{n} ; \mathscr{H}\right) \longrightarrow \bigoplus_{m \geqq 0} L^{2}\left(\boldsymbol{R}^{m} ; \mathscr{K}\right)
$$

(with $F^{[m, n]}: L^{2}\left(\mathbb{R}^{n} ; \mathscr{H}\right) \rightarrow L^{2}\left(\mathbb{R}^{m} ; \mathscr{H}\right)$ ). We have

$$
\begin{aligned}
F^{[m, n]} & =\sum_{l=\max (0, n-m)}^{n} F^{(l-(n-m), 0, l)}+\sum_{l=\max (0, n-m)}^{n-1} F^{(l-(n-m), 1, l)} \\
& =F^{[m-1, n-1]} \otimes 1+F^{(m, 0, n)}+F^{(m-1,1, n-1)} .
\end{aligned}
$$


Here we view $F^{[m-1, n-1]} \otimes 1$ in a canonical way as the operator according to

$$
L^{2}\left(\mathbb{R}^{n} ; \mathscr{H}\right)=L^{2}\left(\mathbb{R}^{n-1} ; \mathscr{H}\right) \otimes L^{2}(\mathbb{R}) \longrightarrow L^{2}\left(\mathbb{R}^{m-1} ; \mathscr{H}\right) \otimes L^{2}(\mathbb{R})=L^{2}\left(\mathbb{R}^{m} ; \mathscr{H}\right) .
$$

Thus $F$ can be written in the form

$$
\left(\begin{array}{ccccc}
F^{(0,0,0)} & F^{(0,0,1)} & F^{(0,0,2)} & F^{(0,0,3)} & \cdots \\
& F^{[0,0]} \otimes 1 & F^{[0,1]} \otimes 1 & F^{[0,2]} \otimes 1 & \\
F^{(1,0,0)} & +F^{(1,0,1)} & +F^{(1,0,2)} & +F^{(1,0,3)} & \cdots \\
& +F^{(0,1,0)} & +F^{(0,1,1)} & +F^{(0,1,2)} & \\
& F^{[1,0]} \otimes 1 & F^{[1,1]} \otimes 1 & F^{[1,2]} \otimes 1 & \\
F^{(2,0,0)} & +F^{(2,0,1)} & +F^{(2,0,2)} & +F^{(2,0,3)} & \cdots \\
& +F^{(1,1,0)} & +F^{(1,1,1)} & +F^{(1,1,2)} & \\
& F^{[2,0]} \otimes 1 & F^{[2,1]} \otimes 1 & F^{[2,2]} \otimes 1 & \\
F^{(3,0,0)} & +F^{(3,0,1)} & +F^{(3,0,2)} & +F^{(3,0,3)} & \ldots \\
& +F^{(2,1,0)} & +F^{(2,1,1)} & +F^{(2,1,2)} & \\
\vdots & \vdots & \vdots & \ddots
\end{array}\right) .
$$

Now $F=0$ implies $F^{[m, n]}=0$ for all $m, n$, thus $F^{(0,0, l)}=0, F^{(n, 0,0)}=0$ and $F^{(n, 1, l)}$ $+F^{(n+1,0, l+1)}=0$ for $n, l \geqq 0$. It remains to show that $F^{(n, 1, l)}$ and $F^{(n+1,0, l+1)}$ vanish singly.

We need an additional argument, which consists mainly in recognizing that $F^{(n+1,0, l+1)}$ acts on the first component as a compact operator, whereas $F^{(n, 1, l)}$ acts as a multiplication operator. For simplicity, we will only treat this for the case $n=2$ and $l=3: F=0$ implies $F^{[2,3]}=F^{[1,2]}=0$, and thus $F^{(2,0,3)}$ $+F^{(1,1,2)}=0$. This implies for almost all $t_{1}, t_{2}$

$$
\begin{aligned}
0= & \left(\left(F^{(2,0,3)}+F^{(1,1,2)}\right) \xi\right)\left(t_{1}, t_{2}\right) \\
= & \iiint f^{(2,0,3)}\left(t_{1}, t_{2} ; 0 ; s_{1}, s_{2}, s_{3}\right) \xi\left(s_{3}, s_{2}, s_{1}\right) d s_{1} d s_{2} d s_{3} \\
& \quad+\iint f^{(1,1,2)}\left(t_{1} ; t_{2} ; s_{1}, s_{2}\right) \xi\left(s_{2}, s_{1}, t_{2}\right) d s_{1} d s_{2}
\end{aligned}
$$

for all $\xi \in L^{2}\left(\mathbb{R}^{3} ; \mathcal{H}\right)$.

For $x, y \in \mathscr{A}$ we define

$$
f_{x, y}^{(n, r, l)}(\sigma ; z ; \nu):=\left\langle x, f^{(n, r, l)}(\sigma ; z ; \nu) y\right\rangle .
$$

Choosing $\xi\left(s_{3}, s_{2}, s_{1}\right)=r_{3}\left(s_{3}\right) r_{2}\left(s_{2}\right) r_{1}\left(s_{1}\right) y$ with $r_{3}, r_{2}, r_{1} \in L^{2}(\boldsymbol{R})$, we get 


$$
\begin{array}{r}
\left.0=\iiint \int f_{x, y}^{(2,0,3)}\left(t_{1}, t_{2} ; \emptyset ; s_{1}, s_{2}, s_{3}\right) r_{1}\left(s_{1}\right) r_{2}\left(s_{2}\right) d s_{1} d s_{2}\right] r_{3}\left(s_{3}\right) d s_{3} \\
+\left[\iint f_{x, y}^{(1,1,2)}\left(t_{1} ; t_{2} ; s_{1}, s_{2}\right) r_{1}\left(s_{1}\right) r_{2}\left(s_{2}\right) d s_{1} d s_{2}\right] r_{3}\left(t_{2}\right)
\end{array}
$$

for almost all $t_{1}, t_{2}$. Let now $v_{1} \in L^{2}(\boldsymbol{R})$. Then we have

$$
\begin{gathered}
\left.0=\iiint \iint f_{x, y}^{(2,0,3)}\left(t_{1}, t_{2} ; \emptyset ; s_{1}, s_{2}, s_{3}\right) r_{1}\left(s_{1}\right) r_{2}\left(s_{2}\right) v_{1}\left(t_{1}\right) d s_{1} d s_{2} d t_{1}\right] r_{3}\left(s_{3}\right) d s_{3} \\
+\left[\iiint f_{x, y}^{(1,1,2)}\left(t_{1} ; t_{2} ; s_{1}, s_{2}\right) r_{1}\left(s_{1}\right) r_{2}\left(s_{2}\right) v_{1}\left(t_{1}\right) d s_{1} d s_{2} d t_{1}\right] r_{3}\left(t_{2}\right)
\end{gathered}
$$

for almost all $t_{2}$. This is now an equation of the form $0=\left(F_{1}+F_{2}\right) r_{3}$, where $F_{1}, F_{2} \in B\left(L^{2}(\boldsymbol{R})\right)$ and $F_{1}$ is a Hilbert-Schmidt-operator, in particular a compact operator, and $F_{2}$ is a multiplication operator. Since a multiplication operator $F_{2} \neq 0$ on $L^{2}(\boldsymbol{R})$ is never compact, we get $F_{1}=F_{2}=0$, i. e. both expressions in the brackets in the above equation are zero for almost all $t_{2}$ and $s_{3}$. Since this is valid for arbitrary $r_{1}, r_{2}, v_{1} \in L^{2}(\boldsymbol{R})$, it follows $\lambda^{2} \times \lambda^{3}$-almost surely that

$$
f_{x, y}^{(2,0,3)}\left(t_{1}, t_{2} ; \emptyset ; s_{1}, s_{2}, s_{3}\right)=\left\langle x, f^{(2,0,3)}\left(t_{1}, t_{2} ; \emptyset ; s_{1}, s_{2}, s_{3}\right) y\right\rangle=0
$$

and $\lambda \times \lambda \times \lambda^{2}$-almost surely that

$$
f_{x, y}^{(1,1,2)}\left(t_{1} ; t_{2} ; s_{1}, s_{2}\right)=\left\langle x, f^{(1,1,2)}\left(t_{1} ; t_{2} ; s_{1}, s_{2}\right) y\right\rangle=0 .
$$

Since $\mathscr{K}$ is assumed to be separable, we can choose a countable basis $B$ of $\mathscr{A}$. Then we have the above equation for all $(x, y) \in B \times B$. This implies:

$$
\begin{aligned}
f^{(2,0,3)}\left(t_{1}, t_{2} ; \emptyset ; s_{1}, s_{2}, s_{3}\right)=0 & \lambda^{2} \times \lambda^{3} \text {-almost surely } \\
f^{(1,1,2)}\left(t_{1} ; t_{2} ; s_{1}, s_{2}\right)=0 & \lambda \times \lambda \times \lambda^{2} \text {-almost surely, }
\end{aligned}
$$

i. e. $f^{(2,0,3)}=0$ and $f^{(1,1,2)}=0$.

Now we want to rewrite multiplication and adjungation for the kernels.

Theorem 2. Let $f, g \in \mathfrak{B}$ be kernels and $F, G \in B(\mathscr{H} \otimes \mathscr{F})$ the corresponding operators. Then we have:

a) $F^{*}$ is given by a kernel $f^{*}$ with

$$
f^{*}(\sigma ; z ; \nu):=f\left(\nu^{*} ; z ; \sigma^{*}\right)^{*} .
$$

b) $F \cdot G$ is given by a kernel $f * g$ with

$$
\begin{aligned}
(f * g)(\sigma ; z ; \nu)= & \sum_{\substack{\nu 1 z_{2} \nu_{2=\nu} \\
\nu_{1} \neq \overline{\bar{g}}}} \int f\left(\sigma ; z ; \nu_{1} \pi\right) g\left(\pi^{*} ; z_{2} ; \nu_{2}\right) d \mu(\pi) \\
& +\sum_{\substack{\sigma_{1} z_{1} \sigma_{2}=\sigma \\
\sigma_{2} \neq \bar{g}}} \int f\left(\sigma_{1} ; z_{1} ; \pi\right) g\left(\pi^{*} \sigma_{2} ; z ; \nu\right) d \mu(\pi) \\
& +\sum_{z_{1} z_{2}=z} \int f\left(\sigma ; z_{1} ; \pi\right) g\left(\pi^{*} ; z_{2} ; \nu\right) d \mu(\pi)
\end{aligned}
$$




\section{Remarks.}

1) We also have $(f * g) *=g * * f *$.

2) The formula for $f * g$ is only a compact notation for the algebraic relations between $p(h), l(g)$ and $l *(f)$. With

$$
f=\bigoplus_{\left(n_{1}, r_{1}, l_{1}\right)} f^{\left(n_{1}, r_{1}, l_{1}\right)} \quad \text { and } \quad g=\bigoplus_{\left(n_{2}, r_{2}, l_{2}\right)} g^{\left(n_{2}, r_{2}, l_{2}\right)}
$$

we have of course

$$
f * g=\sum_{\substack{\left(n_{1}, r_{1}, l_{1}\right) \\\left(n_{2}, r_{2}, l_{2}\right)}} f^{\left(n_{1}, r_{1}, l_{1}\right)} * g^{\left(n_{2}, r_{2}, l_{2}\right)} .
$$

Thus it is sufficient to write down $f^{\left(n_{1}, r_{1}, l_{1}\right)} * g^{\left(n_{2}, r_{2}, l_{2}\right)}$ for all $\left(n_{1}, r_{1}, l_{1}\right)$ and $\left(n_{2}, r_{2}, l_{2}\right)$. There we have to distinguish different cases, according whether the $p$ are absorbed by a $l$ or by a $l^{*}$, or whether two $p$ merge together. This is a little bit costly, but each case is simple. For completeness we give all cases. If $\left(n_{1}, r_{1}, l_{1}\right)$ and $\left(n_{2}, r_{2}, l_{2}\right)$ are given, then also the triple $(n, r, l)$ of $h=f * g$ is determined according to the following diagram.

$$
\begin{array}{llllcl} 
& r_{1} & r_{2} & (n, r, l) & (f * g)(\sigma ; z ; \nu)= \\
l_{1}>n_{2} & 0 & 0 & \left(n_{1}, 0, l_{1}-n_{2}+l_{2}\right) & \int f\left(\sigma ; \emptyset ; \nu_{1} \pi\right) g\left(\pi^{*} ; \emptyset ; \nu_{2}\right) d \mu(\pi) \\
l_{1}>n_{2} & 0 & 1 & \left(n_{1}, 0, l_{1}-n_{2}+l_{2}\right) & \int f\left(\sigma ; \emptyset ; \nu_{1} \pi\right) g\left(\pi^{*} ; \operatorname{end}\left(\nu_{1}\right) ; \nu_{2}\right) d \mu(\pi) \\
l_{1}>n_{2} & 1 & 0 & \left(n_{1}, 1, l_{1}-n_{2}+l_{2}\right) & \int f\left(\sigma ; z ; \nu_{1} \pi\right) g\left(\pi^{*} ; \emptyset ; \nu_{2}\right) d \mu(\pi) \\
l_{1}>n_{2} & 1 & 1 & \left(n_{1}, 1, l_{1}-n_{2}+l_{2}\right) & \int f\left(\sigma ; z ; \nu_{1} \pi\right) g\left(\pi^{*} ; \operatorname{end}\left(\nu_{1}\right) ; \nu_{2}\right) d \mu(\pi) \\
l_{1}<n_{2} & 0 & 0 & \left(n_{1}+n_{2}-l_{1}, 0, l_{2}\right) & \int f\left(\sigma_{1} ; \emptyset ; \pi\right) g\left(\pi^{*} \sigma_{2} ; \emptyset ; \nu\right) d \mu(\pi) \\
l_{1}<n_{2} & 0 & 1 & \left(n_{1}+n_{2}-l_{1}, 1, l_{2}\right) & \int f\left(\sigma_{1} ; \emptyset ; \pi\right) g\left(\pi^{*} \sigma_{2} ; z ; \nu\right) d \mu(\pi) \\
l_{1}<n_{2} & 1 & 0 & \left(n_{1}+n_{2}-l_{1}, 0, l_{2}\right) & \int f\left(\sigma_{1} ; \operatorname{beg}\left(\sigma_{2}\right) ; \pi\right) g\left(\pi^{*} \sigma_{2} ; \emptyset ; \nu\right) d \mu(\pi) \\
l_{1}<n_{2} & 1 & 1 & \left(n_{1}+n_{2}-l_{1}, 1, l_{2}\right) & \int f\left(\sigma_{1} ; \operatorname{beg}\left(\sigma_{2}\right) ; \pi\right) g\left(\pi^{*} \sigma_{2} ; z ; \nu\right) d \mu(\pi) \\
l_{1}=n_{2} & 0 & 0 & \left(n_{1}, 0, l_{2}\right) & \int f(\sigma ; \emptyset ; \pi) g\left(\pi^{*} ; \emptyset ; \nu\right) d \mu(\pi) \\
l_{1}=n_{2} & 0 & 1 & \left(n_{1}, 1, l_{2}\right) & \int f(\sigma ; \emptyset ; \pi) g\left(\pi^{*} ; z ; \nu\right) d \mu(\pi) \\
l_{1}=n_{2} & 1 & 0 & \left(n_{1}, 1, l_{2}\right) & \int f(\sigma ; z ; \pi) g\left(\pi^{*} ; \emptyset ; \nu\right) d \mu(\pi) \\
l_{1}=n_{2} & 1 & 1 & \left(n_{1}, 1, l_{2}\right) & \int f(\sigma ; z ; \pi) g\left(\pi^{*} ; z ; \nu\right) d \mu(\pi) \\
& & & & &
\end{array}
$$


Of course we assume: $\nu_{1} \nu_{2}=\nu, \nu_{1} \neq \emptyset$ and $\sigma_{1} \sigma_{2}=\sigma, \sigma_{2} \neq \emptyset$.

The three terms for $f * g$ in Theorem 2 correspond to the three possibilities $l_{1}>n_{2}, l_{1}<n_{2}$ and $l_{1}=n_{2}$ in the above diagram.

Proof of Theorem 2. a) It is sufficient to show the assertion for fixed $(n, r, l)$. Let $F^{(n, r, l)}$ be the operator corresponding to $f^{(n, r, l)}$ and $H^{(l, r, n)}$ the operator corresponding to $f^{*(n, r, l)}$. We have to show: $H^{(l, r, n)}=F^{(n, r, l) *}$. Take $\xi, \eta \in \mathscr{H} \otimes \mathscr{I}=L^{2}(\Gamma ; \mathscr{H})$. Then we have

$$
\begin{aligned}
& \left\langle F^{(n, r, l)} \xi, \eta\right\rangle_{\mathscr{H} \otimes \mathscr{F}} \\
& =\int\left\langle\left(F^{(n, r, l)} \xi\right)(\omega), \eta(\omega)\right\rangle_{\Re} d \mu(\omega) \\
& =\iiint\left\langle\left(F^{(n, r, l)} \xi\right)\left(\omega_{1} z \omega_{2}\right), \eta\left(\omega_{1} z \omega_{2}\right)\right\rangle_{\mathscr{H}} d \lambda^{n}\left(\omega_{1}\right) d \lambda^{r}(z) d \mu\left(\omega_{2}\right) \\
& =\iiint\left\langle\int f^{(n, r, l)}\left(\omega_{1} ; z ; \nu\right) \xi\left(\nu^{*} z \omega_{2}\right) d \lambda^{l}(\nu), \eta\left(\omega_{1} z \omega_{2}\right)\right\rangle_{\mathscr{H}} d \lambda^{n}\left(\omega_{1}\right) d \lambda^{r}(z) d \mu\left(\omega_{2}\right) \\
& =\iiint \int\left\langle\xi\left(\nu^{*} z \omega_{2}\right), f^{(n, r, l)}\left(\omega_{1} ; z ; \nu\right)^{*} \eta\left(\omega_{1} z \omega_{2}\right)\right\rangle_{\mathscr{T}} d \lambda^{l}(\nu) d \lambda^{n}\left(\omega_{1}\right) d \lambda^{r}(z) d \mu\left(\omega_{2}\right) \\
& =\iiint\left\langle\xi\left(\nu^{*} z \omega_{2}\right), \int f^{*(n, r, l)}\left(\nu^{*} ; z ; \omega_{1}^{*}\right) \eta\left(\omega_{1} z \omega_{2}\right) d \lambda^{n}\left(\omega_{1}\right)\right\rangle_{\mathscr{H}} d \lambda^{l}(\nu) d \lambda^{r}(z) d \mu\left(\omega_{2}\right) \\
& =\iiint\left\langle\xi\left(\nu^{*} z \omega_{2}\right),\left(H^{(l, r, n)} \eta\right)\left(\nu^{*} z \omega_{2}\right)\right\rangle \mathscr{H} d \lambda^{l}(\nu) d \lambda^{r}(z) d \mu\left(\omega_{2}\right) \\
& =\int\left\langle\xi(\omega),\left(H^{(l, r, n)} \eta\right)(\omega)\right\rangle_{\mathscr{H}} d \mu(\omega) \\
& =\left\langle\xi, H^{(l, r, n)} \eta\right\rangle_{\mathfrak{r} \otimes \Phi} \text {. }
\end{aligned}
$$

Since this is valid for all $\xi, \eta \in \mathscr{H} \otimes \mathscr{F}$, the assertion follows.

b) This proof requires the checking of the formula for $f^{\left(n_{1}, r_{1}, l_{1}\right) * g\left(n_{2}, r_{2}, l_{2}\right)}$ for all $\left(n_{1}, r_{1}, l_{1}\right)$ and $\left(n_{2}, r_{2}, l_{2}\right)$. We will only treat the case $l_{1}>n_{2}$. The other two cases are analogous. Let $F, G, H$ be the operators corresponding to $f, g, h$, respectively. The formula for $h:=f * g$ reads in our case as

$$
h^{(n, r, l)}(\sigma ; z ; \nu)=\sum_{\substack{\nu_{1} z_{2} \nu_{2}=\nu \\ \nu 1 \neq \varnothing}} \int f^{\left(n_{1}, r_{1}, l_{1}\right)}\left(\sigma ; z ; \nu_{1} \pi\right) g^{\left(n_{2}, r_{2}, l_{2}\right)}\left(\pi^{*} ; z_{2} ; \nu_{2}\right) d \lambda^{n_{2}(\pi),}
$$

where $l=l_{1}-n_{2}+l_{2}$. We have

$$
\begin{aligned}
& (H \xi)(\omega)=\sum_{\omega_{1} z \omega_{2}=\omega} \int h^{\left(n_{1}, r, l_{1}-n_{2}+l_{2}\right)}\left(\omega_{1} ; z ; \nu\right) \xi\left(\nu^{*} z \omega_{2}\right) d \lambda^{l_{1}-n_{2}+l_{2}(\nu)} \\
& \left.=\sum_{\omega_{1} z \omega_{2}=\omega} \sum_{\substack{\nu_{1} z z_{2}^{2}=\nu \\
\nu_{1} \neq \bar{\phi}}} \iiint f^{\left(n_{1}, r_{1}, l_{1}\right)}\right)\left(\omega_{1} ; z ; \nu_{1} \pi\right) g^{\left(n_{2}, r_{2}, l_{2}\right)}\left(\pi^{*} ; z_{2} ; \nu_{2}\right) \\
& \xi\left(\nu_{2}^{*} z_{2} \nu_{1}^{*} z \omega_{2}\right) d \lambda^{n_{2}}(\pi) d \lambda^{l_{1}-n_{2}}\left(\nu_{1}\right) d \lambda^{l_{2}}\left(\nu_{2}\right)
\end{aligned}
$$




$$
\begin{aligned}
& \stackrel{(*)}{=} \sum_{\omega_{1} z \omega_{2}=\omega} \int f^{\left(n_{1}, r_{1}, l_{1}\right)}\left(\omega_{1} ; z ; \nu_{1} \pi\right)(G \xi)\left(\pi^{*} \nu_{1}^{*} z \omega_{2}\right) d \lambda^{l_{1}}\left(\nu_{1} \pi\right) \\
& =(F G \xi)(\omega) .
\end{aligned}
$$

In $(*)$ we have integrated over $\nu_{2}$ and used the definition of $G$ in terms of $g$. Thus it follows that $H=F \cdot G$.

Theorem 3. We have

and

$$
\left\|f^{*}\right\|_{\mathfrak{B}}=\|f\|_{\mathfrak{B}}
$$

$$
\|f * g\|_{\mathfrak{B}} \leqq\|f\|_{\mathfrak{B}}\|g\|_{\mathfrak{B}},
$$

i.e. $\mathfrak{B}$ with the involution $f \mapsto f *$ and the multiplication $(f, g) \mapsto f * g$ is an involutive Banach algebra.

Proof. The assertion for $f^{*}$ is clear.

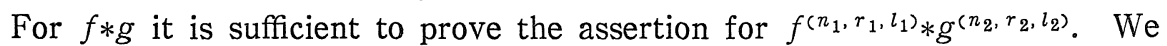
will only do this for $l_{1}>n_{2}$. The other cases are analogous. Let $h:=f * g$. It follows that

$$
\begin{aligned}
& \left\|h^{\left(n_{1}, r, l_{1}-n_{2}+l_{2}\right)}\right\|_{\mathfrak{B}}^{2} \\
& =\sup _{z \in R^{r}} \iint\left\|h^{\left(n_{1}, r, l_{1}-n_{2}+l_{2}\right)}(\sigma ; z ; \nu)\right\|_{\mathfrak{B}(\mathscr{I})}^{2} d \lambda^{n_{1}}(\sigma) d \lambda^{l_{1}-n_{2}+l_{2}(\nu)} \\
& \stackrel{(*)}{=} \sup _{z \in R^{r}} \iiint \int \| \int f^{\left(n_{1}, r_{1}, l_{1}\right)}\left(\sigma ; z ; \nu_{1} z_{2} \pi\right) \\
& g^{\left(n_{2}, r_{2}, l_{2}\right)}\left(\pi^{*} ; z_{2} ; \nu_{2}\right) d \lambda^{n_{2}}(\pi) \|_{B(\mathscr{H})}^{2} d \lambda^{n_{1}}(\boldsymbol{\sigma}) d \lambda^{l_{1}-n_{2}-r_{2}\left(\boldsymbol{\nu}_{1}\right) d \lambda^{r}{ }_{2}\left(z_{2}\right) d \lambda^{l} l_{2}\left(\boldsymbol{\nu}_{2}\right)} \\
& \leqq \sup _{z \in R^{r}} \iiint \int\left[\int\left\|f^{\left(n_{1}, r_{1}, l_{1}\right)}\left(\sigma ; z ; \nu_{1} z_{2} \pi\right)\right\|_{B(\mathscr{g l})}\right.
\end{aligned}
$$

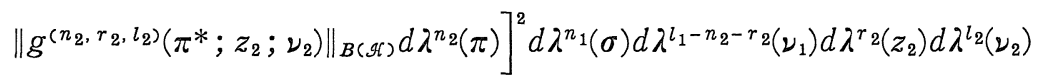

$$
\begin{aligned}
& \stackrel{(* *)}{\leqq} \sup _{z \in R^{r}} \iiint \int\left\{\int\left\|f^{\left(n_{1}, r_{1}, l_{1}\right)}\left(\sigma ; z ; \nu_{1} z_{2} \pi\right)\right\|_{B(\mathscr{F})}^{2} d \lambda^{n_{2}}(\pi)\right\} \\
& \left\{\int\left\|g^{\left(n_{2}, r_{2}, l_{2}\right)}\left(\pi^{*} ; z_{2} ; \nu_{2}\right)\right\|_{B(\mathscr{H})}^{2} d \lambda^{n_{2}(\pi)}\right\} d \lambda^{n_{1}}(\boldsymbol{\sigma}) d \lambda^{l_{1}-n_{2}-r_{2}}\left(\nu_{1}\right) d \lambda^{r_{2}}\left(z_{2}\right) d \lambda^{l_{2}\left(\nu_{2}\right)} \\
& =\sup _{z \in R^{r}} \iint\left\{\iint\left\|f^{\left(n_{1}, r_{1}, l_{1}\right)}\left(\sigma ; z ; \nu_{1} z_{2} \pi\right)\right\|_{B(\mathscr{H})}^{2} d \lambda^{n_{2}}(\pi) d \lambda^{n_{1}}(\sigma)\right\} \\
& \left\{\iint\left\|g^{\left(n_{2}, r_{2}, l_{2}\right)}\left(\pi^{*} ; z_{2} ; \nu_{2}\right)\right\|_{B(\mathscr{H})}^{2} d \lambda^{n_{2}}(\pi) d \lambda^{l_{2}\left(\nu_{2}\right)}\right\} d \lambda^{l_{1}-n_{2}-r_{2}}\left(\nu_{1}\right) d \lambda^{r}{ }^{2}\left(z_{2}\right)
\end{aligned}
$$




$$
\begin{aligned}
& \leqq \\
& \leqq \sup _{z \in R^{r}} \iint\left\|f^{\left(n_{1}, r_{1}, l_{1}\right)}\left(\sigma ; z ; \nu_{1} z_{2} \pi\right)\right\|_{B(\mathscr{H})}^{2} d \lambda^{n_{1}}(\sigma) d \lambda^{\left.l_{1}\left(\nu_{1} z_{2} \pi\right)\right\}} \\
& \quad\left\{\sup _{z^{\prime} \in R} \iint\left\|g^{\left(n_{2}, r_{2}, l_{2}\right)}\left(\pi^{*} ; z^{\prime} ; \nu_{2}\right)\right\|_{B(\mathscr{H})}^{2} d \lambda^{n_{2}}\left(\pi^{*}\right) d \lambda^{l_{2}\left(\nu_{2}\right)}\right\} \\
& =\left\|f^{\left(n_{1}, r_{1}, l_{1}\right)}\right\|_{\mathfrak{B}}^{2}\left\|g^{\left(n_{2}, r_{2}, l_{2}\right)}\right\|_{\mathfrak{B}}^{2} .
\end{aligned}
$$

In $(*)$ we have used the definition of $h=f * g$ and split the integration with respect to $\nu$ into integrations with respect to $\nu_{1}, z_{2}$ and $\nu_{2}$ with $\nu_{=} \nu_{1} z_{2} \nu_{2}$. In $(* *)$ we have used the Cauchy-Schwarz-inequality for the inner integration with respect to $\pi$.

\section{$\S 4$. Definition of Adapted Processes}

Now we introduce processes with values in $\mathfrak{B}$. Again, they will be defined as $l^{1}$-sums of processes with values in $\mathfrak{B}^{(n, r, l)}$. Canonically, we would define the latter as elements of $L^{\infty}\left(K ; \mathfrak{B}^{(n, r, l)}\right)$, where $K$ is a compact subset of $\boldsymbol{R}$. This corresponds to the functions from $K$ to $\mathfrak{B}^{(n, r, l)}$ which can be approximated in the norm $\sup _{t \in K}\left\|f_{t}^{(n, r, l)}\right\|$ by simple functions. According to the remarks at the end of Section 2 this set is too small. Using our kernel representation for $\mathfrak{B}^{(n, r, l)}$ it is reasonable to replace $L^{\infty}\left(K ; \mathfrak{B}^{(n, r, l)}\right)=$ $L^{\infty}\left(K ; L^{\infty}\left(\boldsymbol{R}^{r} ; L^{2}\left(\boldsymbol{R}^{n} \times \boldsymbol{R}^{l} ; B(\mathscr{K})\right)\right)\right)$ by the bigger set

$$
\mathscr{M}^{(n, r, l)}:=L^{\infty}\left(K \times \boldsymbol{R}^{r} ; L^{2}\left(\boldsymbol{R}^{n} \times \boldsymbol{R}^{l} ; B(\mathscr{H})\right)\right) .
$$

Of course, this only makes a difference for $r=1$. This extension is a crucial step, because e. g. the process $F(t)=p(0, t)$ will correspond to the characteristic function on the triangle $A=\{(t, z) \mid 0 \leqq z \leqq t\}$. This characteristic function is an element of $L^{\infty}(K \times \boldsymbol{R})$, but not of $L^{\infty}\left(K ; L^{\infty}(\boldsymbol{R})\right)$.

Furthermore our processes have to be adapted. This reads in the following way.

Definition. We call $f^{(n, r, l)} \in \mathscr{M}^{(n, r, l)}$ adapted, if we have $\lambda \times \lambda^{n} \times \lambda^{r} \times \lambda^{l}$ almost surely :

$$
f_{t}^{(n, r, l)}(\sigma ; z ; \nu)=0 \quad \text { for } \sigma z \nu \not \subset(-\infty, t] .
$$

If we introduce the notations

$$
\begin{aligned}
\mathfrak{B}^{(n, r, l)}(I):= & \left\{f \in \mathfrak{B}^{(n, r, l)} \mid f(\sigma ; z ; \nu)=0\right. \\
& \text { for } \left.\sigma z \nu \not \subset I \lambda^{n} \times \lambda^{r} \times \lambda^{l} \text {-almost surely }\right\} \\
\mathfrak{B}(I):= & \underset{(n, r, l)}{\bigoplus^{l_{1}} \mathfrak{B}^{(n, r, l)}(I),}
\end{aligned}
$$

then the adaptedness of $f$ may be expressed as: For almost all $t$ we have $f_{t} \in \mathfrak{B}(-\infty, t)$. 
As usual it does not matter whether $I$ is open or closed. Thus we can write $\mathfrak{B}\left(t_{1}, t_{2}\right)$ for $\mathfrak{B}\left(\left[t_{1}, t_{2}\right]\right)=\mathfrak{B}\left(\left(t_{1}, t_{2}\right)\right)=\cdots$

Definition. Adapted processes are elements of

$$
\begin{aligned}
\varepsilon: & : \underset{(n, r, l)}{\bigoplus^{l_{1}} \varepsilon^{(n, r, l)}} \\
& =\left\{f=\underset{(n, r, l)}{\bigoplus} f^{(n, r, l)} \mid f^{(n, r, l)} \in \varepsilon^{(n, r, l)} ;\|f\|_{\varepsilon}:=\sum_{(n, r, l)}\left\|f^{(n, r, l)}\right\|_{(n, r, l)}<\infty\right\},
\end{aligned}
$$

where

$$
\boldsymbol{\varepsilon}^{(n, r, l)}:=\left\{f^{(n, r, l)} \in L^{\infty}\left(K \times \mathbb{R}^{r} ; L^{2}\left(\boldsymbol{R}^{n} \times \boldsymbol{R}^{l} ; B(\mathscr{H})\right)\right) \mid f^{(n, r, l)} \text { is adapted }\right\} .
$$

Thus the norm on $\varepsilon^{(n, r, l)}$ is given by

$$
\left\|f^{(n, r, l)}\right\|_{(n, r, l)}^{2}:=\sup _{t \in K} \sup _{z \in R^{r}} \iint\left\|f_{t}^{(n, r, l)}(\sigma ; z ; \nu)\right\|_{B(\mathscr{H})}^{2} d \lambda^{n}(\sigma) d \lambda^{l}(\nu),
$$

where sup denotes as usual the essential supremum with respect to $\lambda$ and $\lambda^{r}$, respectively. As a set we can interpret $\varepsilon$ as a subset of

$$
L^{\infty}\left(K \times \tilde{\boldsymbol{R}} ; L^{2}(\Gamma \times \Gamma ; B(\mathscr{K}))\right) .
$$

\section{Examples.}

1) The process $F(t)=l(0, t)$ corresponds to the function $l=l^{(0,0,1)} \in \varepsilon^{(0,0,1)}$ given by

$$
l_{t}(\emptyset ; \emptyset ; s)= \begin{cases}1, & \text { for } 0 \leqq s \leqq t \\ 0, & \text { else. }\end{cases}
$$

2) The process $F(t)=p(0, t)$ corresponds to the function $p=p^{(0,1,0)} \in \varepsilon^{(0,1,0)}$ given by

$$
p_{t}(\emptyset ; z ; \emptyset)= \begin{cases}1, & \text { for } 0 \leqq z \leqq t \\ 0, & \text { else. }\end{cases}
$$

\section{$\S 5 . \quad$ Stochastic Integrals}

Now we can define the stochastic integrals for all $f, g \in \varepsilon$ in a uniform and simple way.

\section{Definition.}

a) $m:=\int_{T_{0}}^{T_{1}} g_{t} * d l_{t} * f_{t}$ is defined by: $m(\sigma ; z ; \nu)$ is only different from zero for $\max (\sigma z \nu)=\max (\nu)=t$ with $T_{0} \leqq t \leqq T_{1}$. In this case we have with $\nu=\nu_{1} t \nu_{2}$

$$
m\left(\sigma ; z ; \nu_{1} t \nu_{2}\right)=g_{t}\left(\sigma ; z ; \nu_{1}\right) f_{t}\left(\emptyset ; \emptyset ; \nu_{2}\right) .
$$

b) $m:=\int_{T_{0}}^{T_{1}} g_{t} * d l_{t}^{*} * f_{t}$ is defined by: $m(\sigma ; z ; \nu)$ is only different from zero 
for $\max (\sigma z \nu)=\max (\sigma)=t$ with $T_{0} \leqq t \leqq T_{1}$. In this case we have with $\sigma=\sigma_{1} t \sigma_{2}$

$$
m\left(\sigma_{1} t \sigma_{2} ; z ; \nu\right)=g_{t}\left(\sigma_{1} ; \emptyset ; \emptyset\right) f_{t}\left(\sigma_{2} ; z ; \nu\right)
$$

c) $m:=\int_{T_{0}}^{T_{1}} g_{t} * d p_{t} * f_{t}$ is defined by: $m(\sigma ; z ; \nu)$ is only different from zero for $\max (\sigma z \nu)=z \neq \emptyset$ with $T_{0} \leqq z \leqq T_{1}$. In this case we have

$$
m(\sigma ; z ; \nu)=g_{z}(\sigma ; \emptyset ; \emptyset) f_{z}(\emptyset ; \emptyset ; \nu) \text {. }
$$

d) $m:=\int_{T_{0}}^{T_{1}} f_{t} d t$ is defined by

$$
m(\sigma ; z ; \nu)=\int_{T_{0}}^{T_{1}} f_{t}(\sigma ; z ; \nu) d t .
$$

The above definitions are of course to be understood as 'almost surely', i.e. we choose a representative for $f$ and $g$ and then we obtain a representative for $m$. On the arguments where $m$ is not defined uniquely-this happens exactly if $t$ occurs more than once in $\nu$ or $\sigma$, i.e. on hyper surfaces in $\boldsymbol{R}^{n+r+l}$ we set $m=0$. The measure of all these cases is zero, so no difficulties arise.

We have

$$
\int_{T_{0}}^{T_{1}} g_{t} * d l_{t}^{*} * f_{t}=\left(\int_{T_{0}}^{T_{1}} f_{t}^{*} * d l_{t} * g_{t}^{*}\right)^{*} .
$$

Now we define analogously to $[\mathrm{KSp}]$ for $f=\bigoplus_{(n, r, l)} f^{(n, r, l)}$ :

$$
\tilde{f}:=\bigoplus_{l \geqq 0} f^{(0,0, l)} \quad \text { and } \quad \tilde{f}:=\bigoplus_{n \geqq 0} f^{(n, 0,0)} \text {. }
$$

Thus, $\tilde{f}$ is that part of $f$ which contains only annihilation operators, and $\bar{f}$ is that part of $f$ which contains only creation operators.

Because of the adaptedness of $g$ and $f$ we have

$$
\int_{T_{0}}^{T_{1}} g_{t} * d l_{t} * f_{t}=\int_{T_{0}}^{T_{1}} g_{t} * d l_{t} * \tilde{f}_{t}
$$

and

$$
\int_{T_{0}}^{T_{1}} g_{t} * d p_{t} * f_{t}=\int_{T_{0}}^{T_{1}} \bar{g}_{t} * d p_{t} * \tilde{f}_{t}
$$

Furthermore the indices of $f$ and $g$ determine the index of $m$, and we have

$$
\begin{gathered}
\int_{T_{0}}^{T_{1}} h_{t}^{(n, r, l)} d t \in \mathfrak{B}^{(n, r, l)} \\
\int_{T_{0}}^{T_{1}} g_{t}^{(n, r, l)} * d l_{t} * f_{t}^{(0,0, k)} \in \mathfrak{B}^{(n, r, l+1+k)} \\
\int_{T_{0}}^{T_{1}} g_{t}^{(n, 0,0)} * d p_{t} * f_{t}^{(0,0, l)} \in \mathfrak{B}^{(n, 1, l)} .
\end{gathered}
$$


(That the integrals belong to $\mathfrak{B}$ will be shown in Theorem 4.) More generally, we have

$$
\begin{aligned}
\left(\int_{T_{0}}^{T_{1}} h_{t} d t\right)^{(n, r, l)} & =\int_{T_{0}}^{T_{1}} h_{t}^{(n, r, l)} d t \\
\left(\int_{T_{0}}^{T_{1}} g_{t} * d l_{t} * f_{t}\right)^{(n, r, l)} & =\sum_{i=0}^{l-1} \int_{T_{0}}^{T_{1}} g_{t}^{(n, r, i)} * d l_{t} * f_{t}^{(0,0, l-1-i)} \\
\left(\int_{T_{0}}^{T_{1}} g_{t} * d p_{t} * f_{t}\right)^{(n, 0, l)} & =0 \\
\left(\int_{T_{0}}^{T_{1}} g_{t} * d p_{t} * f_{t}\right)^{(n, 1, l)} & =\int_{T_{0}}^{T_{1}} g_{t}^{(n, 0,0)} * d p_{t} * f_{t}^{(0,0, l)} .
\end{aligned}
$$

Remark. It is easy to see that, for simple functions, this definition coincides with the usual definition as given in $[\mathrm{KSp}]$. To see this consider for example

$$
f_{t}=\sum_{i=1}^{k} \chi_{I_{i}}(t) f_{i} \quad \text { and } \quad g_{t}=\sum_{i=1}^{k} \chi_{I_{i}}(t) g_{i}
$$

with $I_{i}=\left[t_{i}, t_{i+1}\right), f_{i}, g_{i} \in \mathfrak{B}\left(-\infty, t_{i}\right)$ and $f_{t}=\tilde{f}_{t}$. Then

$$
m:=\int_{I} g_{t} * d l_{t} * f_{t}
$$

is given by $\left(t=\max \left(\sigma z \nu_{1} t \nu_{2}\right)\right)$

$$
m\left(\sigma ; z ; \nu_{1} t \nu_{2}\right)=\sum_{i=1}^{k} \chi_{I_{i} \cap I}(t) g_{i}\left(\sigma ; z ; \nu_{1}\right) f_{i}\left(\emptyset ; \emptyset ; \nu_{2}\right) .
$$

The corresponding operator $M$ is thus given by

$$
\begin{aligned}
(M \xi)(\omega) & =\sum_{\omega_{1} z \omega_{2}=\omega} \int m\left(\omega_{1} ; z ; \nu\right) \xi\left(\nu^{*} z \omega_{2}\right) d \mu(\nu) \\
& \stackrel{*}{=} \sum_{\omega_{1} z \omega_{2}=\omega} \sum_{i=1}^{k} \iiint \chi_{I_{i} \cap I}(t) g_{i}\left(\omega_{1} ; z ; \nu_{1}\right) f_{i}\left(\emptyset ; \emptyset ; \nu_{2}\right) \\
& \xi\left(\nu_{2}^{*} t \nu_{1}^{*} z \omega_{2}\right) d \mu\left(\nu_{1}\right) d \lambda^{1}(t) d \mu\left(\nu_{2}\right) \\
= & \sum_{i=1}^{k} \sum_{\omega_{1} z \omega_{2}=\omega} \iint g_{i}\left(\omega_{1} ; z ; \nu_{1}\right) \chi_{I_{i} \cap I}(t) \\
& \left.=\sum_{i=1}^{k} \sum_{\omega_{1} z \omega_{2}=\omega} \int g_{i} \xi\right)\left(t \nu_{1}^{*} z \omega_{2}\right) d \lambda^{1}(t) d \mu\left(\omega_{1}\right) \\
& \left(l\left(I_{i} \cap I\right) F_{i} \xi\right)\left(\nu_{1}^{*} z \omega_{2}\right) d \mu\left(\nu_{1}\right) \\
= & \sum_{i=1}^{k}\left(G_{i} l\left(I_{i} \cap I\right) F_{i} \xi\right)(\omega) .
\end{aligned}
$$

There, $F_{i}, G_{i}$ are the operators corresponding to $f_{i}, g_{i}$.

In $(*)$ we have used the definition of $m$ and split the integration over $\nu$ into integrations over $\nu_{1}, t$ and $\nu_{2}$ with $\nu=\nu_{1} t \nu_{2}$. The conditions in the definition of 
$m\left(t=\max \left(\omega_{1} z \nu_{1} t \nu_{2}\right)\right)$ are automatically satisfied because of the adaptedness of $g_{i}$ and $f_{i}$.

Theorem 4. Let $f, g, h \in \varepsilon$ and define

$$
m^{1}:=\int_{0}^{T} h_{t} d t, \quad m^{2}:=\int_{0}^{T} g_{t} * d l_{t} * f_{t}, \quad m^{3}:=\int_{0}^{T} g_{t} * d p_{t} * f_{t} .
$$

Then $m^{1}, m^{2}, m^{3} \in \mathfrak{B}(-\infty, T)$, and we have:

$$
\begin{aligned}
& \left\|m^{1}\right\|_{\mathfrak{B}} \leqq T\|h\|_{\mathcal{\varepsilon}} \\
& \left\|m^{2}\right\|_{\mathfrak{B}} \leqq \sqrt{T}\|g\|_{\varepsilon}\|\tilde{f}\|_{\varepsilon} \\
& \left\|m^{3}\right\|_{\mathfrak{B}} \leqq\|\bar{g}\|_{\varepsilon}\|\tilde{f}\|_{\varepsilon} .
\end{aligned}
$$

Proof. a) Adaptedness is clear from the definition of the integrals.

b) We have

$$
\begin{aligned}
& \left\|m^{1(n, r, l)}\right\|^{2}=\sup _{z \in R^{r}} \iint\left\|m^{1(n, r, l)}(\sigma ; z ; \nu)\right\|_{B(\mathscr{H})}^{2} d \lambda^{n}(\boldsymbol{\sigma}) d \lambda^{l}(\nu) \\
& =\sup _{z \in R^{r}} \iint\left\|\int_{0}^{T} h_{t}^{(n, r, l)}(\sigma ; z ; \nu) d t\right\|_{B(\mathscr{H})}^{2} d \lambda^{n}(\sigma) d \lambda^{l}(\nu) \\
& \leqq \sup _{z \in \boldsymbol{R}^{r}} \iint\left[\int_{0}^{T}\left\|h_{t}^{(n, r, l)}(\sigma ; z ; \nu)\right\|_{B(\mathscr{H})} d t\right]^{2} d \lambda^{n}(\sigma) d \lambda^{l}(\nu) \\
& \stackrel{(*)}{\leqq} \sup _{z \in R^{r}} \iint\left\{T \int_{0}^{T}\left\|h_{t}^{(n, r, l)}(\sigma ; z ; \nu)\right\|_{B(\mathscr{H})}^{2} d t\right\} d \lambda^{n}(\boldsymbol{\sigma}) d \lambda^{l}(\boldsymbol{\nu}) \\
& \leqq T \sup _{z \in \boldsymbol{R}^{T}} \int_{0}^{T} \sup _{s \in K} \iint\left\|h_{s}^{(n, r, l)}(\sigma ; z ; \boldsymbol{\nu})\right\|_{B(\mathscr{H})}^{2} d \lambda^{n}(\boldsymbol{\sigma}) d \lambda^{l}(\boldsymbol{\nu}) d t \\
& =T^{2}\left\|h^{(n, r, l)}\right\|_{(n, r, l)}^{2} \text {. }
\end{aligned}
$$

In $(*)$ we have used the Cauchy-Schwarz-inequality for $\chi_{(0, T)} \cdot h^{(n, r, l)}$.

Thus it follows that

c) We have

$$
\begin{aligned}
\left\|m^{1}\right\|_{\mathfrak{B}} & =\sum_{(n, r, l)}\left\|m^{1(n, r, l)}\right\| \\
& \leqq T \sum_{(n, r, l)}\left\|h^{(n, r, l)}\right\|_{(n, r, l)} \\
& =T\|h\|_{\varepsilon} .
\end{aligned}
$$

$$
m^{2(n, r, l)}=\sum_{i=0}^{l-1} \int_{0}^{T} g_{t}^{(n, r, i)} * d l_{t} * f_{t}^{(0,0, l-1-i)} .
$$

First we consider 


$$
\begin{aligned}
& \left\|\int_{0}^{T} g_{t}^{(n, r, i)} * d l_{t} * f_{t}^{(0,0, l-1-i)}\right\|^{2} \\
& =\sup _{z \in R^{r}} \iint\left\|\left(\int_{0}^{T} g_{t}^{(n, r, i)} * d l_{t} * f_{t}^{(0,0, l-1-i)}\right)(\sigma ; z ; \nu)\right\|_{B(\mathscr{H})}^{2} d \lambda^{n}(\sigma) d \lambda^{l}(\nu) \\
& \stackrel{(*)}{=} \sup _{z \in R^{r}} \iint_{0}^{T} \iint\left\|g_{s}^{(n, r, i)}\left(\sigma ; z ; \nu_{1}\right) f_{s}^{(0,0, l-1-i)}\left(\emptyset ; \emptyset ; \nu_{2}\right)\right\|_{B(\mathscr{H})}^{2} \\
& d \lambda^{n}(\sigma) d \lambda^{i}\left(\nu_{1}\right) d \lambda(s) d \lambda^{l-1-i}\left(\nu_{2}\right) \\
& \leqq \int_{0}^{T} \sup _{z \in R^{r}} \iiint\left\|g_{s}^{(n, r, i)}\left(\sigma ; z ; \nu_{1}\right)\right\|_{B(\mathscr{H})}^{2} \\
& \left\|f_{s}^{(0,0, l-1-i)}\left(\emptyset ; \emptyset ; \nu_{2}\right)\right\|_{B(\mathscr{H})}^{2} d \lambda^{n}(\sigma) d \lambda^{i}\left(\nu_{1}\right) d \lambda^{l-1-i}\left(\nu_{2}\right) d \lambda(s) \\
& \leqq \int_{0}^{T}\left\{\sup _{z \in R^{r}} \iint\left\|g_{s}^{(n, r, i)}\left(\sigma ; z ; \nu_{1}\right)\right\|_{B(\mathscr{H})}^{2} d \lambda^{n}(\sigma) d \lambda^{i}\left(\nu_{1}\right)\right\} \\
& \left\{\int\left\|f_{s}^{(0,0, l-1-i)}\left(\emptyset ; \emptyset ; \nu_{2}\right)\right\|_{B(\mathscr{H})}^{2} d \lambda^{l-1-i}\left(\nu_{2}\right)\right\} d \lambda(s) \\
& =\int_{0}^{T}\left\|g_{s}^{(n, r, i)}\right\|^{2}\left\|f_{s}^{(0,0, l-1-i)}\right\|^{2} d \lambda(s) \\
& \leqq \int_{0}^{T} \sup _{t \in K}\left\|g_{t}^{(n, r, i)}\right\|^{2} \sup _{t^{\prime} \in K}\left\|f_{t^{\prime}}^{(0,0, l-1-i)}\right\|^{2} d \lambda(s) \\
& =T\left\|g^{(n, r, i)}\right\|_{(n, r, i)}^{2}\left\|f^{(0,0, l-1-i)}\right\|_{(0,0, l-1-i)}^{2} .
\end{aligned}
$$

In $(*)$ we have used the definition of the stochastic integral with respect to $d l_{t}$ and then split $\nu$ into $\nu_{1} S \nu_{2}$.

Thus it follows that

$$
\begin{aligned}
\left\|m^{2}\right\|_{\mathfrak{B}} & =\sum_{(n, r, l)}\left\|m^{2(n, r, l)}\right\| \\
& \leqq \sum_{(n, r, l)} \sum_{i=0}^{l-1} \sqrt{T}\left\|g^{(n, r, i)}\right\|_{(n, r, i)}\left\|f^{(0,0, l-1-i)}\right\|_{(0,0, l-1-i)} \\
& =\sqrt{T}\|g\|_{\varepsilon}\|\tilde{f}\|_{\varepsilon} .
\end{aligned}
$$

d) We have $m^{(n, 0, l)}=0$ and

$$
m^{(n, 1, l)}=\int_{0}^{T} g_{t}^{(n, 0,0)} * d p_{t} * f_{t}^{(0,0, l)} .
$$

First we consider

$$
\begin{aligned}
& \left\|\int_{0}^{T} g_{t}^{(n, 0,0)} * d p_{t} * f_{i}^{(0,0, l)}\right\|^{2} \\
& \quad=\sup _{z \in R} \iint\left\|\left(\int_{0}^{T} g_{t}^{(n, 0,0)} * d p_{t} * f_{t}^{(0,0, l)}\right)(\sigma ; z ; \nu)\right\|_{B(\mathcal{H})}^{2} d \lambda^{n}(\sigma) d \lambda^{l}(\nu)
\end{aligned}
$$




$$
\begin{aligned}
& =\sup _{z \in[0, T]} \iint\left\|g_{z}^{(n, 0,0)}(\sigma ; \emptyset ; \emptyset) f_{z}^{(0,0, l)}(\emptyset ; \emptyset ; \nu)\right\|_{B(\mathscr{H})}^{2} d \lambda^{n}(\sigma) d \lambda^{l}(\nu) \\
& \leqq\left\|g^{(n, 0,0)}\right\|_{(n, 0,0)}^{2}\left\|f^{(0,0, l)}\right\|_{(0,0, l)}^{2} .
\end{aligned}
$$

(compare part c)

Thus it follows that

$$
\begin{aligned}
\left\|m^{3}\right\|_{\mathfrak{B}} & =\sum_{(n, 1, l)}\left\|m^{3(n, 1, l)}\right\| \\
& \leqq \sum_{n, l \geqq 0}\left\|g^{(n, 0,0)}\right\|_{(n, 0,0)}\left\|f^{(0,0, l)}\right\|_{(0,0, l)} \\
& =\|\bar{g}\|_{\varepsilon}\|\tilde{f}\|_{\varepsilon} .
\end{aligned}
$$

e) It remains to show that all stochastic integrals are measurable functions (Bochner integrability includes measurability). It is sufficient to see this for fixed indices of $f, g$ and $h$. To see this, consider first special $f, g, h$ : Let them be functions with countable range from $L^{\infty}\left(K \times \boldsymbol{R}^{r} ; L^{2}\left(\boldsymbol{R}^{n} \times \boldsymbol{R}^{l} ; B(\mathscr{H})\right)\right)$ and $L^{\infty}\left(K \times \boldsymbol{R}^{0} ; L^{2}\left(\boldsymbol{R}^{0} \times \boldsymbol{R}^{k} ; B(\mathscr{C})\right)\right)$, respectively, i.e.

$$
\begin{aligned}
& g_{t}^{(n, r, l)}(\sigma ; z ; \nu)=\sum_{i=1}^{\infty} \chi_{A_{i}}(t, z) g^{i}(\sigma ; \nu) \\
& f_{t}^{(0,0, k)}(\emptyset ; \emptyset ; \nu)=\sum_{i=1}^{\infty} \chi_{B_{i}}(t, \emptyset) f^{i}(\emptyset ; \nu) \\
& h_{t}^{(n, r, l)}(\sigma ; z ; \nu)=\sum_{i=1}^{\infty} \chi_{C_{i}}(t, z) h^{i}(\sigma ; \nu)
\end{aligned}
$$

with $A_{i}, C_{i} \in \mathfrak{L}\left(K \times \boldsymbol{R}^{r}\right), \quad B_{i} \in \mathfrak{L}\left(K \times \boldsymbol{R}^{0}\right), \quad g^{i}, h^{i} \in L^{2}\left(\boldsymbol{R}^{n} \times \boldsymbol{R}^{l} ; B(\mathscr{H})\right) \quad$ and $\quad f_{\imath} \in$ $L^{2}\left(\boldsymbol{R}^{0} \times \boldsymbol{R}^{k} ; B(\mathscr{H})\right)$. Then we have

$$
\begin{aligned}
m^{1(n, r, l)}(\sigma ; z ; \nu)= & \sum_{i=1}^{\infty} \int_{0}^{T} \chi_{C_{i}}(t, z) d t h^{i}(\sigma ; \nu), \\
m^{2(n, r, l+1+k)}(\sigma ; z ; \nu)= & \sum_{i, j=1}^{\infty} \chi_{A_{i}}(\max (\nu), z) g^{i}\left(\sigma ; \nu_{1}\right) \chi_{B_{j}}(\max (\nu), \emptyset) f^{j}\left(\emptyset ; \nu_{2}\right) \\
& \chi_{(0, T)}(\max (\nu)) \chi_{(-\infty, \max (\nu))}(\max (\sigma)) \chi_{(-\infty, \max (\nu))}(z)
\end{aligned}
$$

(with $\left.\nu=\nu_{1} \max (\nu) \nu_{2}\right)$ and

$$
\begin{aligned}
m^{3(n, 1, k)}(\sigma ; z ; \nu)= & \sum_{i, j=1}^{\infty} \chi_{A_{i}}(z, \emptyset) g^{i}(\sigma ; \emptyset) \chi_{B_{j}}(z, \emptyset) f^{j}(\emptyset ; \nu) \\
& \chi_{(0, T)}(z) \chi_{(-\infty, z)}(\max (\sigma)) \chi_{(-\infty, z)}(\max (\nu)) .
\end{aligned}
$$

These three functions (regarded as functions in $z$ ) are measurable and are thus elements of $\mathfrak{B}$. (For example, for $m^{1}$ this is true, because $z \mapsto \int_{0}^{T} \chi_{C_{i}}(t, z) d t$ is a measurable function.)

Since $f, g, h$ of the above form are dense in $\varepsilon$, the assertion for general 
$f, g, h \in \varepsilon$ follows from the shown norm inequalities.

\section{§6. Ito's Formula}

In the following we put $K=[0, T]$. Given processes $f, g, h \in \varepsilon$ we can define new processes by

$$
\begin{aligned}
& m_{t}^{1}:=\int_{0}^{t} h_{s} d s \\
& m_{t}^{2}:=\int_{0}^{t} g_{s} * d l_{s} * f_{s} \\
& m_{t}^{3}:=\int_{0}^{t} g_{s} * d p_{s} * f_{s} .
\end{aligned}
$$

Theorem 5. If $f, g, h \in \varepsilon$, then $m^{1}, m^{2}, m^{3} \in \varepsilon$, and we have

$$
\begin{aligned}
& \left\|m^{1}\right\|_{\varepsilon} \leqq T\|h\|_{\varepsilon} \\
& \left\|m^{2}\right\|_{\varepsilon} \leqq \sqrt{T}\|g\|_{\varepsilon}\|\tilde{f}\|_{\varepsilon} \\
& \left\|m^{3}\right\|_{\varepsilon} \leqq\|\bar{g}\|_{\varepsilon}\|\tilde{f}\|_{\varepsilon} .
\end{aligned}
$$

Proof. The inequalities follow directly from Theorem 4.

It remains to show that $m^{1}, m^{2}, m^{3}$ are measurable functions. But this is clear if we look again at the form of these integrals for $f, g, h$ with countable range (part e) of the proof of Theorem 4, replace $T$ by $t$ ): For example, $\chi_{(0, t)}(z)$ is a simple function in $(t, z)$ (but not in $t$ alone!), and $\int_{0}^{t} \chi_{C_{i}}(s, z) d s$ and $\chi_{(0, t)}(\max (\nu))$ can be approximated by simple functions.

The differential equation

$$
(*)\left\{\begin{array}{l}
d m_{t}=g_{t}^{1} * d p_{t} * f_{t}^{1}+g_{t}^{2} * d l_{t} * f_{t}^{2}+g_{t}^{3} * d l_{t}^{*} * f_{t}^{3}+h_{t} d t \\
m_{0}=m_{a}
\end{array}\right.
$$

(with $m_{a} \in \mathfrak{B}(-\infty, 0)$ ) is now a formal notation for the validity for almost all $t \in K$ of

$$
m_{t}=m_{a}+\int_{0}^{t} g_{s}^{1} * d p_{s} * f_{s}^{1}+\int_{0}^{t} g_{s}^{2} * d l_{s} * f_{s}^{2}+\int_{0}^{t} g_{s}^{3} * d l_{s}^{*} * f_{s}^{3}+\int_{0}^{t} h_{s} d s
$$

Theorem 6. Consider $f^{i}, g^{i}, h \in \varepsilon(i=1,2,3)$. The process $m \in \varepsilon$ is a solution of $(*)$ if and only if the following conditions are fulfilled for almost all $(t ; \sigma ; z ; \nu)$ :

i) For $r=\max (\sigma r \nu)$ with $0 \leqq r \leqq t$ we have 


$$
m_{t}(\sigma ; r ; \nu)=m_{a}(\sigma ; r ; \nu)+g_{r}^{1}(\sigma ; \emptyset ; \emptyset) f_{r}^{1}(\emptyset ; \emptyset ; \nu)+\int_{r}^{t} h_{s}(\sigma ; r ; \nu) d s .
$$

ii) For $r=\max \left(\sigma z \nu_{1} r \nu_{2}\right)$ with $0 \leqq r \leqq t$ we have

$$
\begin{aligned}
& m_{t}\left(\sigma ; z ; \nu_{1} r \nu_{2}\right)=m_{a}\left(\sigma ; z ; \nu_{1} r \nu_{2}\right)+g_{r}^{2}\left(\sigma ; z ; \nu_{1}\right) f_{r}^{2}\left(\emptyset ; \emptyset ; \nu_{2}\right) \\
& \quad+\int_{r}^{t} h_{s}\left(\sigma ; z ; \nu_{1} r \nu_{2}\right) d s .
\end{aligned}
$$

iii) For $r=\max \left(\sigma_{1} r \sigma_{2} z \nu\right)$ with $0 \leqq r \leqq t$ we have

$$
\begin{aligned}
& m_{t}\left(\sigma_{1} r \sigma_{2} ; z ; \nu\right)=m_{a}\left(\sigma_{1} r \sigma_{2} ; z ; \nu\right)+g_{r}^{3}\left(\sigma_{1} ; \emptyset ; \emptyset\right) f_{r}^{3}\left(\sigma_{2} ; z ; \nu\right) \\
&+\int_{r}^{t} h_{s}\left(\sigma_{1} r \sigma_{2} ; z ; \nu\right) d s .
\end{aligned}
$$

iv) For $\max (\sigma z \nu)<0$ we have $m_{t}(\sigma ; z ; \nu)=m_{a}(\sigma ; z ; \nu)$.

Proof. The assertion follows directly from the definition of the stochastic integrals.

Theorem 7 (Ito's Fomula). For $e, f, g, h \in \varepsilon$ consider

$$
\begin{aligned}
& m_{t}^{1}:=\int_{0}^{t} g_{s} * d \bar{l}_{s}^{1} * f_{s} \\
& m_{t}^{2}:=\int_{0}^{t} e_{s} * d \tilde{l}_{s}^{2} * h_{s},
\end{aligned}
$$

where $d \bar{l}_{s}^{1}$ and $d \bar{l}_{s}^{2}$ denote any of the four symbols $d p_{s}, d l_{s}, d l_{s}^{*}$ and $d s$. Define $m_{t}:=m_{t}^{1} * m_{t}^{2}$. Then we have for all $t \in K$

$$
m_{t}=\int_{0}^{t} g_{s} * d \bar{l}_{s}^{1} * f_{s} * m_{s}^{2}+\int_{0}^{t} m_{s}^{1} * e_{s} * d \bar{l}_{s}^{2} * h_{s}+\int_{0}^{t} g_{s} *\left(f_{s} * e_{s}\right)^{(0,0,0)} * d \bar{l}_{s} * h_{s},
$$

where $d \bar{l}_{s}$ is given by the following diagram:

$\begin{array}{ccccc}d \bar{l}_{s}^{1} \backslash d \bar{l}_{s}^{2} & d p_{s} & d l_{s}^{*} & d l_{s} & d s \\ d p_{s} & d p_{s} & d l_{s}^{*} & 0 & 0 \\ d l_{s}^{*} & 0 & 0 & 0 & 0 \\ d l_{s} & d l_{s} & d s & 0 & 0 \\ d s & 0 & 0 & 0 & 0\end{array}$

Proof. Since the definitions of multiplication and stochastic integration are explicity given, we only have to put the definitions of all expressions into Ito's formula and compare the two sides of the equation. Thus the assertion follows from a straightforward calculation.

On the other hand, these calculations are very tedious because we have to distinguish many cases. For illustration we will here present only the short 
computation for $d \bar{l}_{s}^{1}=d p_{s}$ and $d \bar{l}_{s}^{2}=d p_{s}$. Let $z=\max (\sigma z \nu)$ with $0 \leqq z \leqq t$. Then we have

$$
\begin{aligned}
& \left(m_{t}^{1} * m_{t}^{2}\right)(\sigma ; z ; \nu) \\
& =\sum_{\substack{\nu_{1} z z_{2}^{2}=\bar{\nu}=\nu \\
\nu_{1} \neq \bar{\emptyset}}} \int m_{t}^{1}\left(\sigma ; z ; \nu_{1} \pi\right) m_{t}^{2}\left(\pi^{*} ; z_{2} ; \nu_{2}\right) d \mu(\pi) \\
& +\sum_{\substack{\sigma_{1} z \sigma_{2}=\sigma \\
\sigma_{2} \neq \bar{\emptyset}}} \int m_{t}^{1}\left(\sigma_{1} ; z_{1} ; \pi\right) m_{t}^{2}\left(\pi * \sigma_{2} ; z ; \nu\right) d \mu(\pi) \\
& +\int m_{t}^{1}(\sigma ; z ; \pi) m_{t}^{2}\left(\pi^{*} ; \emptyset ; \nu\right) d \mu(\pi) \\
& +\int m_{t}^{1}(\sigma ; \emptyset ; \pi) m_{t}^{2}\left(\pi^{*} ; z ; \nu\right) d \mu(\pi) \\
& +\int m_{t}^{1}(\sigma ; z ; \pi) m_{t}^{2}\left(\pi^{*} ; z ; \nu\right) d \mu(\pi) \\
& =\sum_{\substack{\nu_{1} z_{2} \nu_{2}=\nu \\
\nu_{1} \neq \emptyset}} \int g_{z}(\sigma ; \emptyset ; \emptyset) f_{z}\left(\emptyset ; \emptyset ; \nu_{1} \pi\right) m_{z}^{2}\left(\pi^{*} ; z_{2} ; \nu_{2}\right) d \mu(\pi) \\
& +\sum_{\substack{\sigma_{1} z_{2} \sigma_{2}=\sigma \\
\sigma_{2} \neq \emptyset}} \int m_{z}^{1}\left(\sigma_{1} ; z_{1} ; \pi\right) e_{z}\left(\pi^{*} \sigma_{2} ; \emptyset ; \emptyset\right) h_{z}(\emptyset ; \emptyset ; \nu) d \mu(\pi) \\
& +\int g_{z}(\sigma ; \emptyset ; \emptyset) f_{z}(\emptyset ; \emptyset ; \pi) m_{z}^{2}\left(\pi^{*} ; \emptyset ; \nu\right) d \mu(\pi) \\
& +\int m_{z}^{1}(\sigma ; \emptyset ; \pi) e_{z}\left(\pi^{*} ; \emptyset ; \emptyset\right) h_{z}(\emptyset ; \emptyset ; \nu) d \mu(\pi) \\
& +\int g_{z}(\sigma ; \emptyset ; \emptyset) f_{z}(\emptyset ; \emptyset ; \pi) e_{z}\left(\pi^{*} ; \emptyset ; \emptyset\right) h_{z}(\emptyset ; \emptyset ; \nu) d \mu(\pi) \\
& =g_{z}(\sigma ; \emptyset ; \emptyset) \cdot\left(f_{z} * m_{z}^{2}\right)(\emptyset ; \emptyset ; \nu)+\left(m_{z}^{1} * e_{z}\right)(\sigma ; \emptyset ; \emptyset) \cdot h_{z}(\emptyset ; \emptyset ; \nu) \\
& +g_{z}(\sigma ; \emptyset ; \emptyset) \cdot\left(f_{z} * e_{z}\right)(\emptyset ; \emptyset ; \emptyset) \cdot h_{z}(\emptyset ; \emptyset ; \nu) \\
& =\left[\int_{0}^{t} g_{s} * d p_{s} * f_{s} * m_{s}^{2}+\int_{0}^{t} m_{s}^{1} * e_{s} * d p_{s} * h_{s}\right. \\
& \left.+\int_{0}^{t} g_{s} *\left(f_{s} * e_{s}\right)^{(0,0,0)} * d p_{s} * h_{s}\right](\sigma ; z ; \nu) .
\end{aligned}
$$

One sees directly the vanishing of the expressions corresponding to Theorem 6 ii), iii) and iv). This gives the assertion.

\section{§7. Stochastic Differential Equations}

For constructing stochastic evolutions we have to consider linear stochastic differential equations of the form 


$$
\text { (*) }\left\{\begin{array}{l}
d v_{t}=v_{t} *\left\{g_{t}^{0} * d p_{t} * f_{t}^{0}+g_{t}^{1} * d l_{t} * f_{t}^{1}+g_{t}^{2} * d l_{t}^{*} * f_{t}^{2}+h_{t} d t\right\} \\
v_{0}=v_{a} \in \mathfrak{B}(-\infty, 0)
\end{array}\right.
$$

The mere transfer of the corresponding existence and uniqueness theorem of [KSp] is wrecked on the fact that in the estimate for the integral with respect to $d p$ (Theorem 5) the length of the integration interval does not appear. The following example shows that this is a serious problem.

Example. Let $\mathscr{H}=\boldsymbol{C}$. We are looking for the solution of the stochastic differential equation

$$
(* *) \quad\left\{\begin{array}{l}
d v_{t}=v_{t} * g_{t} * d p_{t} \\
v_{0}=\mathrm{id}
\end{array}\right.
$$

For $g$ we choose the process $g=g^{(1,0,0)}$ with

$$
g_{t}^{(1,0,0)}\left(t_{1} ; \emptyset ; \emptyset\right)=\frac{1}{\sqrt{t}} \chi_{(0, t)}\left(t_{1}\right) .
$$

Then $g$ is an element of $\varepsilon^{(1,0,0)}$. The kernel $g_{t}$ corresponds to the operator $(1 / \sqrt{t}) l^{*}(0, t)$ which has, independently of $t$, the norm 1 .

Thus we are looking for $v \in \varepsilon$ with

$$
v_{t}=\mathrm{id}+\int_{0}^{t} v_{s} * g_{s}^{(1,0,0)} * d p_{s} .
$$

Let $v_{t}=\bigoplus_{(n, r, l)} v_{t}^{(n, r, l)}$. Then it is easy to see that (note $v_{t}^{(n, 0,1)}=0$ )

$$
\begin{aligned}
& v_{t}^{(0,0,0)}=\mathrm{id}=\mathrm{id}^{(0,0,0)} \\
& v_{t}^{(1,1,0)}=\int_{0}^{t} v_{s}^{(0,0,0)} * g_{s}^{(1,0,0)} * d p_{s} \\
& v_{t}^{(n, 1,0)}=\int_{0}^{t} v_{s}^{(n-1,1,0)} * g_{s}^{(1,0,0)} * d p_{s} \quad(n \geqq 2) .
\end{aligned}
$$

This can be solved iteratively and we find

$$
\begin{aligned}
v_{t}^{(0,0,0)}(\emptyset ; \emptyset ; \emptyset) & =1 \\
v_{t}^{(1,1,0)}\left(t_{1} ; z ; \emptyset\right) & = \begin{cases}\frac{1}{\sqrt{z}} & 0 \leqq t_{1} \leqq z \leqq t \\
0 & \text { else }\end{cases} \\
v_{t}^{(n, 1,0)}\left(t_{1}, \cdots, t_{n} ; z ; \emptyset\right) & =\left\{\begin{array}{cl}
\frac{1}{\sqrt{z t_{n} t_{n-1} \cdots t_{3} t_{2}}} & 0 \leqq t_{1} \leqq \cdots \leqq t_{n} \leqq z \leqq t \\
0 & \text { else }
\end{array}\right.
\end{aligned}
$$

But now we have 


$$
\begin{aligned}
\left\|v^{(n, 1,0)}\right\|_{(n, 1,0)}^{2} & =\sup _{t \in[0, T]} \sup _{z \in R} \int \cdots \int\left|v_{t}^{(n, 1,0)}\left(t_{1}, \cdots, t_{n} ; z ; \emptyset\right)\right|^{2} d t_{1} \cdots d t_{n} \\
& =\sup _{t \in[0, T]} \sup _{z \in R} \int_{0}^{z} \cdots \int_{0}^{t_{3}} \int_{0}^{t_{2}} \frac{1}{z} \frac{1}{t_{n}} \frac{1}{t_{n-1}} \cdots \frac{1}{t_{2}} d t_{1} d t_{2} \cdots d t_{n} \\
& =1,
\end{aligned}
$$

because $\int_{0}^{t_{2}} d t_{1}=t_{2}$ etc.

Thus, $\left\|v^{(n, 1,0)}\right\|_{(n, 1,0)}=1$, and in the same way $\left\|v^{(0,0,0)}\right\|_{(0,0,0)}=1$ and $\left\|v^{(1,1,0)}\right\|_{(1,1,0)}$ $=1$. (By the way, the other $v^{(n, r, l)}$ vanish.) This means

$$
\|v\|_{\varepsilon} \geqq \sum_{n=2}^{\infty}\left\|v^{(n, 1,0)}\right\|=\infty,
$$

i.e. (**) has no solution in $\varepsilon$.

Luckily, for our dilations we will only need the special case of $(*)$, where $f_{t}^{i}, g_{t}^{i}, h_{t}(i=0,1,2)$ are operators in $B(\mathscr{K})$, i.e. where they are elements of $\varepsilon^{(0,0,0)}$. In this situation we have again an existence and uniqueness theorem, because in this case the integration with respect to $d p$ can only appear once in the iteration procedure.

Theorem 8. The stochastic differential equation

$$
\left\{\begin{array}{l}
d v_{t}=v_{t} *\left\{g_{t}^{0} * d p_{t} * f_{t}^{0}+g_{t}^{1} * d l_{t} * f_{t}^{1}+g_{t}^{2} * d l_{t}^{*} * f_{t}^{2}+h_{t} d t\right\} \\
v_{0}=v_{a} \in \mathfrak{B}(-\infty, 0)
\end{array}\right.
$$

has a unique solution $v \in \varepsilon$, if $f^{0}, f^{1}, f^{2}, g^{1}, g^{2}, h \in \varepsilon$ and $g^{0}=g^{0(0,0,0)} \in \varepsilon^{(0,0,0)}$.

Proof. As usual we try an iteration procedure on $\left[0, t_{0}\right]: v_{t}^{1} \equiv v_{a}$ and

$$
\begin{aligned}
v_{t}^{r}=v_{a}+\int_{0}^{t} v_{s}^{r-1} * g_{s}^{0} * d p_{s} & f_{s}^{0}+\int_{0}^{t} v_{s}^{r-1} * g_{s}^{1} * d l_{s} * f_{s}^{1} \\
& +\int_{0}^{t} v_{s}^{r-1} * g_{s}^{2} * d l_{s}^{*} * f_{s}^{2}+\int_{0}^{t} v_{s}^{r-1} * h_{s} d s
\end{aligned}
$$

$(r \geqq 2)$. We get

$$
\begin{aligned}
\left\|v^{r}-v^{r-1}\right\|_{\varepsilon} \leqq & \left\|\overline{v^{r-1} * g^{0}}-\overline{v^{r-2} * g^{0}}\right\|_{\varepsilon}\left\|\tilde{f}^{0}\right\|_{\varepsilon} \\
& +\left\|v^{r-1}-v^{r-2}\right\|_{\varepsilon}\left\{\sqrt{t_{0}}\left\|g^{1}\right\|_{\varepsilon}\left\|f^{1}\right\|_{\varepsilon}+\sqrt{t_{0}}\left\|g^{2}\right\|_{\varepsilon}\left\|f^{2}\right\|_{\varepsilon}+t_{0}\|h\|_{\varepsilon}\right\} .
\end{aligned}
$$

Since $g^{0}=g^{0(0,0,0)}$, we now have

$$
\overline{v^{r-1} * g^{0}}=\sum_{n \geqq 0}\left(v^{r-1} * g^{0}\right)^{(n, 0,0)}=\sum_{n \geqq 0} v^{r-1(n, 0,0)} * g^{0(0,0,0)}=\overline{v^{r-1}} * g^{0} .
$$

We use 


$$
\overline{v_{t}^{r-1}}=\overline{v_{a}}+\int_{0}^{t} \overline{v_{s}^{r-2} * g_{s}^{2} *} d l_{s}^{*} * \overline{f_{s}^{2}}+\int_{0}^{t} \overline{v_{s}^{r-2} * h_{s}} d s
$$

(integration with respect to $d p$ can only appear once) to conclude

$$
\begin{aligned}
\overline{\| v^{r-1} * g^{0}}-\overline{v^{r-2} * g^{0}} \|_{\varepsilon} & \leqq \overline{v^{r-1}}-\overline{v^{r-2}}\left\|_{\varepsilon}\right\| g^{0} \|_{\varepsilon} \\
& \leqq\left\|v^{r-2}-v^{r-3}\right\|_{\varepsilon}\left\{\sqrt{t_{0}}\left\|g^{2}\right\|_{\varepsilon}\left\|f^{2}\right\|_{\varepsilon}+t_{0}\|h\|_{\varepsilon}\right\}\left\|g^{0}\right\|_{\varepsilon} .
\end{aligned}
$$

Together with the above estimate for $\left\|v^{r}-v^{r-1}\right\|_{s}$ this guarantees the applicability of Banach's ixpoint theorem (for double iteration), and thus the assertion follows.

Remariss. 1) We may choose a cancnical representative of $v$ in the following way: Define

$$
v_{t}=v_{a}+\int_{0}^{t} a v_{s}
$$

for every $t \in K$. The right side of this definition does not depend on the choice of the representative.

2) Contrary to the situation in [KSp], the canonical representative may be discontinuous. For erample, the solution of

$$
\left\{\begin{array}{l}
d v_{t}=v_{t} * d p_{t} \\
v_{0}=\mathrm{id}
\end{array}\right.
$$

is given by $v_{t}=\mathrm{id}+p_{t}$, which corresponds to $V(t)=1+p(0, t)$. This process has no (uniformly) continuous representative.

\section{§8. Siveincstic Evolutioris}

Next, we triy to find conditions which guarantee unitarity of the solution of our stochastic differential equation. Again we restrict to a special class of differential equations, namely

$$
(*) \quad\left\{\begin{array}{l}
d u_{t}=u_{t} *\left\{e_{t} * d p_{t}+g_{t} * d l_{t}+d l_{t}^{*} * f_{t}+h_{t} d t\right\} \\
u_{0}=\mathrm{id}
\end{array}\right.
$$

where $f, g, h \in \varepsilon$ and $e=e^{(0,0,0)} \in \varepsilon^{(0,0,0)}$.

Note for the following calculations that elements of $\varepsilon^{(0,0,0)}$ commute with all differentials (e.g. $e_{t} * d p_{t}=d p_{t} * e_{t}=d p_{t} e_{t}$ ).

By the differential equation for $u^{*}$

$$
\left\{\begin{array}{l}
d u_{t}^{*}=\left\{d p_{t} * e_{t}^{*}+d l_{t}^{*} * g_{i}^{*}+f_{t}^{*} * d l_{t}+h_{t}^{*} d t\right\} * u_{t}^{*} \\
u_{v}^{*}=\mathrm{id}
\end{array}\right.
$$


and by Ito's formula, we find as a necessary condition for unitarity of $u_{t}$

$$
\begin{aligned}
0= & d\left(u_{t}^{*} * u_{t}\right) \\
= & d u_{t}^{*} * u_{t}+u_{t}^{*} * d u_{t}+d u_{t}^{*} * d u_{t} \\
= & d p_{t} *\left[e_{t}^{*}+e_{t}+e_{t}^{*} * e_{t}\right]+d l_{t}^{*} *\left[g_{t}^{*}+f_{t}+e_{t}^{*} * f_{t}\right] \\
& +\left[f_{t}^{*}+g_{t}+f_{t}^{*} * e_{t}\right] * d l+\left[h_{t}^{*}+h_{t}+f_{t}^{*} * f_{t}\right] d t .
\end{aligned}
$$

The next lemma enables us to conclude the vanishing of the coefficients of the single differentials.

Lemma 1. Consider $a, b, c, d, e, f, h \in \varepsilon$ and

$$
d m_{t}:=a_{t} * d p_{t} * b_{t}+c_{t} * d l_{t}^{*} * d_{t}+e_{t} * d l_{t} * f_{t}+h_{t} d t .
$$

a) The following conditions are equivalent:

i) $d m_{t}=0$.

ii) For almost all $\left(t ; \sigma ; \sigma_{1} ; \sigma_{2} ; z ; \nu ; \nu_{1} ; \nu_{2}\right)$ we have

$$
\begin{array}{r}
a_{t}(\sigma ; \emptyset ; \emptyset) b_{t}(\emptyset ; \emptyset ; \nu)=0 \\
c_{t}\left(\sigma_{1} ; \emptyset ; \emptyset\right) d_{t}\left(\sigma_{2} ; z ; \nu\right)=0 \\
e_{t}\left(\sigma ; z ; \nu_{1}\right) f_{t}\left(\emptyset ; \emptyset ; \nu_{2}\right)=0 \\
h_{t}(\sigma ; z ; \nu)=0 .
\end{array}
$$

b) In particular, $d m_{t}=0$ implies for almost every $t$ that

$$
\begin{aligned}
\bar{a}_{t} * \tilde{b}_{t} & =0 \\
\bar{c}_{t} * d_{t} & =0 \\
e_{t} * \tilde{f}_{t} & =0 \\
h_{t} & =0 .
\end{aligned}
$$

Proof. a) By the definition of the stochastic integrals, the implication ii) $\Rightarrow$ i) is clear.

For the reverse implication we consider

$$
n_{t}=\int_{0}^{t} a_{s} * d p_{s} * b_{s}+\int_{0}^{t} c_{s} * d l_{s}^{*} * d_{s}+\int_{0}^{t} e_{s} * d l_{s} * f_{s}+\int_{0}^{t} h_{s} d s .
$$

According to the assumption we have $m_{t}=0$ for all $t$. The following considerations are valid almost surely.

For $(\sigma ; z ; \nu)$ with $z=\max (\sigma z \nu)$ and $z \leqq t$ we have

$$
0=m_{t}(\sigma ; z ; \nu)=a_{z}(\sigma ; \emptyset ; \emptyset) b_{z}(\emptyset ; \emptyset ; \nu)+\int_{z}^{t} h_{s}(\sigma ; z ; \nu) d s .
$$


For $t \downarrow z$ this implies

i.e. the first assertion.

$$
0=a_{z}(\sigma ; \emptyset ; \emptyset) b_{z}(\emptyset ; \emptyset ; \nu),
$$

For $(\sigma ; z ; \nu)$ with $\sigma=\sigma_{1} r \sigma_{2}, r=\max (\sigma z \nu)$ and $r \leqq t$ we have

$$
0=m_{t}\left(\sigma_{1} r \sigma_{2} ; z ; \nu\right)=c_{r}\left(\sigma_{1} ; \emptyset ; \emptyset\right) d_{r}\left(\sigma_{2} ; z ; \nu\right)+\int_{r}^{t} h_{s}(\sigma ; z ; \nu) d s .
$$

For $t \downarrow r$ this implies

$$
0=c_{r}\left(\sigma_{1} ; \emptyset ; \emptyset\right) d_{r}\left(\sigma_{2} ; z ; \nu\right),
$$

i.e. the second assertion.

The third assertion is proved in the same way.

Furthermore we have

$$
\left(m_{t_{2}}-m_{t_{1}}\right)(\sigma ; z ; \nu)=\int_{t_{1}}^{t_{2}} h_{s}(\sigma ; z ; \nu) d s=0 \quad \text { for } t_{2}>t_{1} \geqq \max (\sigma z \nu) .
$$

Because of the adaptedness of $h$ we have for all $t_{1}, t_{2} \in K$

$$
\int_{t_{1}}^{t_{2}} h_{s}(\sigma ; z ; \nu) d s=\int_{t_{1}}^{\max (\sigma ; z ; \nu)} h_{s}(\sigma ; z ; \nu) d s+\int_{\max (\sigma ; z ; \nu)}^{t_{2}} h_{s}(\sigma ; z ; \nu) d s=0,
$$

i.e. $h_{t}(\sigma ; z ; \nu)=0$ almost everywhere.

b) This follows from a):

$$
\left(\bar{c}_{t} * d_{t}\right)(\sigma ; z ; \nu)=\sum_{\sigma_{1} \sigma_{2}=\sigma} c_{t}\left(\sigma_{1} ; \emptyset ; \emptyset\right) d_{t}\left(\sigma_{2} ; z ; \nu\right)=0
$$

and in the same way for $\bar{a}_{t} * \tilde{b}_{t}$ and $e_{t} * \tilde{f}_{t}$.

This lemma gives us the following three necessary conditions for unitarity:

$$
\begin{aligned}
& e_{t}^{*}+e_{t}+e_{t}^{*} * e_{t}=0 \\
& g_{t}^{*}+f_{t}+e_{t}^{*} * f_{t}=0 \\
& h_{t}^{*}+h_{t}+f_{t}^{*} * f_{t}=0 .
\end{aligned}
$$

Contrary to the case without $d p\left(e_{t}=0\right)$, where these conditions are sufficient, $u_{t} * u_{t}^{*}=\mathrm{id}$ gives us another condition. Ito's formula implies

$$
\begin{aligned}
0= & d\left(u_{t} * u_{t}^{*}\right) \\
= & u_{t} *\left\{\left[e_{t}+e_{t}^{*}+e_{t} * e_{t}^{*}\right] * d p_{t}+d l_{t}^{*} *\left[f_{t}+g_{t}^{*}+e_{t} * g_{t}^{*}\right]\right. \\
& \left.+\left[g_{t}+f_{t}^{*}+g_{t} * e_{t}^{*}\right] * d l_{t}+\left[h_{t}+h_{t}^{*}+g_{t} * g_{t}^{*}\right] d t\right\} * u_{t}^{*} .
\end{aligned}
$$

We use lemma 1a) (for $d p_{t}$ ) to conclude (note $e_{t} \in \varepsilon^{(0,0,0)}$ )

$$
\begin{aligned}
0 & =\left(u_{t}\left(e_{t}+e_{t}^{*}+e_{t} * e_{t}^{*}\right)\right)(\sigma ; \emptyset ; \emptyset) \cdot u_{t}^{*}(\emptyset ; \emptyset ; \nu) \\
& =u_{t}(\sigma ; \emptyset ; \emptyset) \cdot\left(e_{t}+e_{t}^{*}+e_{t} * e_{t}^{*}\right)(\emptyset ; \emptyset ; \emptyset) \cdot u_{t}^{*}(\emptyset ; \emptyset ; \nu) .
\end{aligned}
$$


Multiplying this on the left by $u_{t}^{*}\left(\emptyset ; \emptyset ; \sigma^{*}\right)$ and on the right by $u_{t}\left(\nu^{*} ; \emptyset ; \emptyset\right)$, integrating over $\sigma$ and $\nu$ and considering

$$
\begin{aligned}
\int u_{t}^{*}(\emptyset ; \emptyset ; \nu) u_{t}\left(\nu^{*} ; \emptyset ; \emptyset\right) d \mu(\nu) & =\left(u_{t}^{*} * u_{t}\right)(\emptyset ; \emptyset ; \emptyset) \\
& =\operatorname{id}(\emptyset ; \emptyset ; \emptyset) \\
& =1_{B(\mathscr{I})}
\end{aligned}
$$

we conclude $e_{t}+e_{t}^{*}+e_{t} * e_{t}^{*}=0$.

Thus we have the following necessary conditions

$$
\begin{aligned}
& e_{t}+e_{t}^{*}+e_{t} * e_{t}^{*}=0 \\
& e_{t}+e_{t}^{*}+e_{t}^{*} * e_{t}=0 \\
& f_{t}+g_{t}^{*}+e_{t}^{*} * f_{t}=0 \\
& h_{t}+h_{t}^{*}+f_{t}^{*} * f_{t}=0 .
\end{aligned}
$$

The first two equations are equivalent to $e_{t}=w_{t}-\mathrm{id}$, where $w_{t}$ is unitary. Then the other two equations give $f_{t}=-w_{t} * g_{t}^{*}$ and $h_{t}=i h_{t}^{s}-(1 / 2) g_{t} * g_{t}^{*}$ with $h_{t}^{s}$ selfadjoint. These conditions are also sufficient.

Theorem 9. Let $g, h^{s} \in \varepsilon$ and $w \in \varepsilon^{(0,0,0)}$, and let for almost every the kernels $w_{t}$ be unitary and $h_{t}^{s}$ selfadjoint. Then the solution $u_{t}$ of

$$
\left\{\begin{array}{l}
d u_{t}=u_{t} *\left\{\left(w_{t}-\mathrm{id}\right) * d p_{t}+g_{t} * d l_{t}-d l_{t}^{*} * w_{t} * g_{t}^{*}+\left(i h_{t}^{s}-\frac{1}{2} g_{t} * g_{t}^{*}\right) d t\right\} \\
u_{0}=\mathrm{id}
\end{array}\right.
$$

is unitary for every $t$.

The statement ' $u_{t}$ is unitary for every $t$ ' means: The canonical representative of $u \in \varepsilon$ is unitary for every $t$.

Proof. The assumptions imply at once that $u_{t} * u_{t}^{*}=\mathrm{id}$ for every $t$. (Note here the validity of Ito's formula for every $t$.) For $w_{t}:=u_{t}^{*} * u_{t}$ we deduce the following differential equation (with $\left.f_{t}=-w_{t} * g_{t}^{*}, h_{t}=i h_{t}^{s}-(1 / 2) g_{t} * g_{t}^{*}\right)$ :

$$
\left\{\begin{aligned}
d w_{t}= & \left\{d p_{t} * e_{t}^{*}+d l_{t}^{*} * g_{t}^{*}+f_{t}^{*} * d l_{t}+h_{t}^{*} d t\right\} * w_{t} \\
& +w_{t} *\left\{e_{t} * d p_{t}+g_{t} * d l_{t}+d l_{t}^{*} * f_{t}+h_{t} d t\right\} \\
& +e_{t}^{*} * w_{t}^{(0,0,0)} * e_{t} * d p_{t}+e_{t}^{*} * w_{t}^{(0,0,0)} * d l_{t}^{*} * f_{t} \\
& +f_{t}^{*} * w_{t}^{(0,0,0)} * e_{t} * d l_{t}+f_{t}^{*} * w_{t}^{(0,0,0)} * f_{t} d t
\end{aligned}\right.
$$

A small extension of the proof of Theorem 8 shows that the solution of this stochastic differential equation is unique. Since $w_{t}=\mathrm{id}$ is a solution, we find 
$w_{t}=u_{t}^{*} * u_{t}=$ id for every $t$.

Example. For $g_{t}=h_{t}^{s}=0$ and $w_{t}=W$.id with unitary $W \in B(\mathscr{K})$ we have the differential equation

$$
\left\{\begin{array}{l}
d u_{t}=u_{t} * d p_{t} \cdot(V-1) \\
u_{0}=\mathrm{id}
\end{array}\right.
$$

The solution is given by $\iota_{t}=\mathrm{id}+(W-1) p_{t}$, which corresponds to $U(t)=$ $1+(W-1) p(0, t)$.

\section{§9. Dilations}

With the help of our extended stochastic calculus we are now able to give additional dilations of the completely positive semigroup $T_{t}=e^{t \mathfrak{R}}$ on $B(\mathscr{K})$. The generator $\mathbb{\sim}$ shall have the form

$$
\begin{aligned}
\Omega: B(\mathscr{H}) & \longrightarrow B(\mathcal{H}) \\
X & \longrightarrow i \llbracket H, X]-\frac{1}{2}\left(L * L X-2 L * X L+X L^{*} L\right),
\end{aligned}
$$

where $H, L \in B(\mathcal{H})$ and $H$ is selfadjoint. By $S_{t}: \mathscr{I} \mapsto \mathscr{I}$ we denote the shift on s- the Fock space. (compare [Lbl, HuP1, KSp])

Theorem 10. Let $u_{t} \in \varepsilon$ be ihe solution of the stochastic differential equaiion

$$
\left\{\begin{array}{l}
d u_{t}=u_{t} *\left\{(W-1) d p_{t}-L * W d l_{t}+d l_{t}^{*} L+\left(i H-\frac{1}{2} L * L\right) d t\right\} \\
u_{0}=\mathrm{id}
\end{array}\right.
$$

where $W \in B(\mathscr{H})$ is unitary. Let $U(t)$ be the unitary operator on $\mathscr{H} \otimes \mathscr{I}$ corresponding to $u_{t}$ and let $\hat{T}_{t}$ for $t \geqq 0$ be given by

$$
\begin{aligned}
\hat{T}_{t}: B(\mathscr{H}) \otimes B(\mathscr{I}) & \longrightarrow B(\mathscr{H}) \otimes B(\mathscr{F}) \\
Z & \longmapsto U(t) S_{t}^{-1} Z S_{t} U^{*}(t) .
\end{aligned}
$$

Then $\hat{T}_{t}$ is a dilation of $T_{t}$, i.e. the following diagram commutes:

where

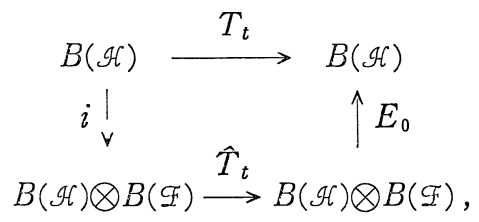

$$
\begin{aligned}
i: B(\mathscr{H}) & \longrightarrow B(\mathscr{H}) \otimes B(\mathscr{I}) \\
X & \longrightarrow X \otimes 1
\end{aligned}
$$

and 


$$
\begin{aligned}
E_{0}: B(\mathscr{K}) \otimes B(\mathscr{F}) & \longrightarrow B(\mathscr{K}) \\
L \otimes A & \longmapsto\langle\Omega, A \Omega\rangle L .
\end{aligned}
$$

Proof. The proof is analogous to the proof of Theorem 7 in $[\mathrm{KSp}]$. $\diamond$

Note that we have not demanded that our dilation be stationary, i.e. we have not addressed the question whether there exists a state which is invariant under $\hat{T}_{t}$. (cf. [FGo, FMa, Küm1, Maa2])

Finally, we want to give explicit solutions for the differential equations appearing in Theorem 10. More generally, we consider differential equations of the form

$$
\text { (*) }\left\{\begin{array}{l}
d v_{t}=v_{t} *\left\{d p_{t} P+d l_{t} R+d l_{t}^{*} S+d t T\right\} \\
v_{0}=\mathrm{id}
\end{array}\right.
$$

with $P, R, S, T \in B(\mathscr{K})$. The analogous equations on the bosonic Fock space were treated by Maassen [Maa1].

Definition. The $n$-tuple $\sigma=\left(t_{1}, t_{2}, \cdots, t_{n}\right)$ is called time-ordered, if we have $t_{1} \leqq t_{2} \leqq \cdots \leqq t_{n}$.

Theorem 11. Let $P, R, S, T \in B(\mathscr{H})$. Then the solution $v \in \varepsilon$ of (*) has the following form:

i) $\sigma z \nu$ not time-ordered: $v_{t}(\sigma ; z ; \nu)=0$.

ii) $\min (\sigma ; z ; \nu)<0: v_{t}(\sigma ; z ; \nu)=0$.

iii) $\sigma z \nu=\left(t_{1}, \cdots, t_{n}\right)$ time-ordered and $0 \leqq t_{1} \leqq \cdots \leqq t_{n} \leqq t$ :

$$
v_{t}(\sigma ; z ; \nu)=e^{t_{1} T} W_{1} e^{\left(t_{2}-t_{1}\right) T} W_{2} \cdots W_{n-1} e^{\left(t_{n}-t_{n-1}\right) T} W_{n} e^{\left(t-t_{n}\right) T},
$$

where

$$
W_{i}= \begin{cases}P & \text { if } t_{i}=z \\ R & \text { if } t_{i} \in \nu \\ S & \text { if } t_{i} \in \sigma\end{cases}
$$

Proof. The equation $(*)$ is equivalent to

$$
v_{t}=\mathrm{id}+\int_{0}^{t} v_{s} * d p_{s} P+\int_{0}^{t} v_{s} * d l_{s} R+\int_{0}^{t} v_{s} * d l_{s}^{*} S+\int_{0}^{t} v_{s} d s T .
$$

Since the solution is unique, we only have to check whether the given $v$ is a solution. We will verify the conditions given in Theorem 6 .

If $\sigma z \nu$ is not time-ordered or if $\min (\sigma z \nu)<0$, then it is easy to see that these conditions are fulfilled.

Consider now $\sigma z \nu=\left(t_{1}, \cdots, t_{n}\right)$ with $0 \leqq t_{1} \leqq \cdots \leqq t_{n} \leqq t$. We only check the condition ii) of Theorem 6 , i.e. we assume $t_{n} \in \nu$. Then we have to show that $\left(\nu=\nu_{1} t_{n}\right)$ 


$$
v_{t}\left(\sigma ; z ; \nu_{1} t_{n}\right)=v_{t_{n}}\left(\sigma ; z ; \nu_{1}\right) R+\int_{t_{n}}^{t} v_{s}(\sigma ; z ; \nu) d s T .
$$

This is valid, because the left side is equal to

$$
v_{t}\left(\sigma ; z ; \nu_{1} t_{n}\right)=v_{t_{n}}\left(\sigma ; z ; \nu_{1}\right) R \cdot e^{\left(t-t_{n}\right) T}
$$

and the right side is equal to

$$
\begin{aligned}
v_{t_{n}}\left(\sigma ; z ; \nu_{1}\right) R+\int_{t_{n}}^{t} v_{s}\left(\sigma ; z ; \nu_{1} t_{n}\right) d s T \\
\quad=v_{t_{n}}\left(\sigma ; z ; \nu_{1}\right) R+v_{t_{n}}\left(\sigma ; z ; \nu_{1}\right) R \int_{t_{n}}^{t} e^{\left(s-t_{n}\right) T} T d s \\
\quad=v_{t_{n}}\left(\sigma ; z ; \nu_{1}\right) R\left[1_{B(\mathscr{H})}+\left[e^{\left(s-t_{n}\right) T}\right]_{t_{n}}^{t}\right] \\
\quad=v_{t_{n}}\left(\sigma ; z ; \nu_{1}\right) R \cdot e^{\left(t-t_{n}\right) T} .
\end{aligned}
$$

The other conditions of Theorem 6 can be verified in the same way.

\section{References}

[AFL] Accardi, L., Frigerio, A. and Lewis, J.T., Quantum stochastic processes, Publ. RIMS, Kyoto Univ., 18 (1982), 97-133.

[ApH] Applebaum, D. B. and Hudson, R.L., Fermion Ito's formula and stochastic evolutions, Commun. Math. Phys., 96 (1984), 473-496.

[Arn] Arnold, L., On Wigner's semicircle law for the eigenvalues of random matrices, Z. Wahrscheinlichkeitstheorie verw. Geb., 19 (1971), 191-198.

[BSW1] Barnett, C., Streater, R.F. and Wilde, I. F., The Ito-Clifford-integral, J. Funct. Anal., 48 (1982), 172-212.

[BSW2] Barnett, C., Streater, R.F. and Wilde, I.F., Quasi-free quantum stochastic integrals for the CAR and CCR, J. Funct. Anal., 52 (1983), 19-47.

[Cun] Cuntz, J., Simple $C^{*}$-algebras generated by isometries, Commun. Math. Phys., 57 (1977), 173-185.

[Dav] Davies, E. B., Markovian master equations, Commun. Math. Phys., 39 (1974), 91-110.

[DiU] Diestel, J. and Uhl, J.J., Vector Measures, (American Mathematical Society, Providence, Rhode Island, 1977).

[Düm] Dümcke, R., Convergence of multi-time correlation functions in the weak and singular coupling limits, J. Math. Phys., 24 (1983), 311-315.

[Eva] Evans, D.E., On $O_{n}$, Publ. RIMS Kyoto Univ., 16 (1980), 915-927.

[FGo] Frigerio, A. and Gorini, V., Markov dilations and quantum detailed balance, Commun. Math. Phys., 93 (1984), 517-532.

[FMa] Frigerio, A. and Maassen, H., Quantum Poisson processes and dilations of dynamical semigroups, Probab. Th. Rel. Fields, 83 (1989), 489-508.

[GaC] Gardiner, C. W. and Collet, M. J., Input and output in damped quantum systems: Quantum stochastic differential equations and the master equation, Phys. Rev. A, 31 (1985), 3761-3774.

[GSS] Glockner, P., Schürmann, M. and Speicher, R., Realization of free white noises, SFB-preprint 564.

[HuP1] Hudson, R.L. and Parthasarathy, K.R., Quantum Ito's formula and stochastic 
evolutions, Commun. Math. Piny's., 23 (1984), 301-323.

[HuP2] Hudson, R.L. and Parthasarathy, K.R., Unification of boson and fermion scochastic calculus, Commun. Math. Phys., 104 (1986), 457-470.

[KSp] Kümmerer, B. and Speicher, R., Stochastic integration on the Cuntz algebra $O_{\infty}$, Preprint, Heidelberg 1990.

[Küm1] Kümmerer, B., Markov dilations on $W^{*}$-algebras, J. Funct. Anal., G3 (1985), $139-177$.

[Küm2] - Survey on a theory of non-commutative stationary Markov processes, in Quanturn Probability and Applications III, Oberwolfach 1987, (Lecture Notes in Mathematics 1203, Springer Verlag, Heidelberg 1988), 154-182.

[Küm3] - Markov dilations and non-commutative Poisson processes, Preprint.

[Lbl] Lindblad, G., On the generators of quantum dynamical semigroups, Commun. Math. Phys., 48 (1976), 119-130.

[LiP] Lindsay, J.M. and Parthasarachy, K.R., Cohomology of power sers with applications in quantum probability, Commun. Math. Phys., 124 (1989), 337-364.

[Mal] Maassen, H., Quantum Markov processes on Fock space described by integral kernels, in Quantum Probability and Applicaitons II, Heidelberg 1984, (Lecture Notes in Mathematics 1136, Springer Verlag, Heidelberg 1985), 361-374.

[Maa2] Maassen, H., Theoretical concepts in quantum probability; quantum Markov processes, Preprint, Nijmegen 1988.

[PSi] Parthasarathy, K.R. and Sinha, K.B., Unification of quantum noise processes in Fock spaces, Preprint, New Delhi 1990.

[Sch] Schürmann, M., Quantum stochastic processes wirh independent addicive increments, Preprint, Heidelberg 1939.

[Spe1] Speicher, R., Quantenstochastische Prozesse auf der Cuntz-Algebra, Thesis, Heidelberg 1989.

[Spe2] —- A New Example of 'Independence' and 'White Noise', Probab. Th. Rel. Fields, 84 (1990), 141-159.

[Voil] Voiculescu, D., Symmetries of some reduced free product $C^{*}$-algebras, in Operaior Algebras and their Conneciion with Topology and Ergodic Theory, Busteni, Romania, 1983, (Leccure Notes in Mathematics 1132, Springer Verlag, Heidelberg 1985).

[Voi2] - Limit laws for random matrices and free products, Preprint IHES M/89/21, Bures-sur-Yvette 1989.

[Vig] Wigner, E.P., Random matrices, SIAM Rev., 9 (1967), 1-23. 